\title{
Dez anos do informe brasileiro sobre espécies exóticas invasoras: avanços, lacunas e direções futuras
}

\author{
Rafael Dudeque Zenni ${ }^{1 *}$ \\ Michele de Sá Dechoum ${ }^{2,3}$ \\ Sílvia Renate Ziller $^{3}$ \\ ${ }^{1}$ Universidade de Brasília, Instituto de Ciências Biológicas, Departamento de Ecologia \\ Campus Darcy Ribeiro, Asa Norte, CEP 70910-900, Brasília - DF, Brasil \\ ${ }^{2}$ Universidade Federal de Santa Catarina, Florianópolis - SC, Brasil \\ ${ }^{3}$ Instituto Hórus de Desenvolvimento e Conservação Ambiental, Florianópolis - SC, Brasil \\ * Autor para correspondência \\ rafaeldz@gmail.com
}

Submetido em 01/10/2015

Aceito para publicação em 27/11/2015

\section{Resumo}

Em 2004, o Ministério do Meio Ambiente contratou a elaboração do Informe Nacional sobre Espécies Exóticas Invasoras, cujos objetivos eram a coleta, a sistematização e a divulgação de informações no Brasil. Neste artigo, revisamos o que existia de conhecimento científico e normativas legais sobre invasões biológicas no Brasil antes do Informe, fazemos uma avaliação dos avanços e lacunas nos 10 anos pós-Informe e sugerimos direções futuras. Encontramos 143 artigos científicos e 65 marcos legais sobre espécies exóticas invasoras publicados entre 1999 e 2014. Os dados indicam que o Informe Nacional representa um divisor de águas para o tema no Brasil, a partir do qual aumentou a frequência da publicação de normas legais e pesquisas científicas. Identificamos avanços, ainda que aquém do necessário para considerar o tema consolidado no país. Os avanços obtidos se devem em parte à mobilização do terceiro setor e a grupos de pesquisa trabalhando separadamente. Para os próximos anos devem ser promovidos projetos de pesquisa, manejo e gestão mais ambiciosos e relevantes para fazer avançar o tema no país, contribuir para seu avanço em termos globais, embasar ações práticas de manejo e consolidar um arcabouço legal integrado que efetivamente permita-nos prevenir, manejar e controlar invasões biológicas.

Palavras-chave: Biologia de invasões; Ecologia de invasões; Informe nacional sobre espécies exóticas invasoras; Legislação; Políticas públicas

\section{Abstract}

Ten years after the Brazilian survey on invasive alien species: advances, gaps, and future directions. In 2004, the Brazilian Ministry of Environment issued a call for projects for a national survey on invasive alien species aimed at collecting, organizing and disseminating information on invasive alien species in Brazil. In this paper we review the existing scientific knowledge and legal regulations on biological invasions in Brazil prior to the National Survey, the progress made since then, the gaps persisting ten years later, and make recommendations for future work. We found 143 scientific papers and 65 legal regulations on invasive alien species published between 1999 and 2014. Data indicate the National Survey is a milestone on the subject in 
Brazil, as the publication of regulations and articles increased significantly after 2005. Although numerous advances were identified, they are not sufficient for the topic to be considered consolidated in Brazil. Advances made were greatly due to the mobilization of civil society organizations and research groups working separately. More ambitious and relevant research and management projects are required in upcoming years to ensure the advancement of invasion science in Brazil in ways that may also contribute internationally. Finally, consolidating management practices and actions into an integrated legal framework is key to effectively enable the prevention, management and control of biological invasions.

Key words: Brazilian survey of invasive alien species; Invasion biology; Invasion ecology; Legislation; Public policy

\section{Introdução}

Invasões biológicas causam mudanças ambientais globais, assim como mudanças climáticas e a conversão direta de ambientes naturais para uso intensivo. Muitas vezes, os efeitos negativos desses fatores são sinérgicos e de grande severidade para sistemas naturais (DIDHAM et al., 2007). Quando invadem ambientes naturais, espécies exóticas podem reduzir a biodiversidade (WINTER et al., 2009; GILBERT; LEVINE, 2013), alterar processos ecossistêmicos (VILÀ et al., 2011; KATSANEVAKIS et al., 2014) e trazer prejuízos socioeconômicos (REASER et al., 2007). No Brasil, os primeiros registros de invasões biológicas datam de 1824, com a observação da expansão populacional gradativa do capim africano Melinis minutiflora $\mathrm{P}$. Beauv. (ZENNI; ZILLER, 2011). O Brasil é citado no livro The Ecology of Invasions by Animals and Plants, publicado por Charles Elton em 1958, como palco de um esforço conjunto entre o governo brasileiro e a Fundação Rockefeller dos Estados Unidos para a erradicação do mosquito africano Anopheles gambiae (Giles 1902), transmissor da malária, no nordeste do país em 1940. O trabalho mobilizou cerca de 3000 pessoas e custou US\$ 2 milhões de dólares (ELTON, 1958). Durante as décadas posteriores, pouco se estudou ou fez sobre espécies exóticas invasoras no Brasil.

No início da década de 1980, liderados pelo Comitê Científico para Problemas Ambientais (SCOPE - Scientific Committee on Problems of the Environment), esforços científicos foram iniciados em diferentes partes do mundo com o intuito de compreender invasões biológicas (DRAKE et al., 1989). Os resultados desses esforços tiveram enorme repercussão e o conhecimento gerado sobre impactos de espécies exóticas invasoras incidiu sobre a agenda conservacionista, culminando com a inclusão do tema na Convenção sobre Diversidade Biológica (CDB) em 1992. Além do Brasil, outros 167 países ratificaram a $\mathrm{CDB}$ naquela data. Atualmente são 196 países. O texto da CDB foi legalizado no Brasil por meio do Decreto Legislativo no 2 , promulgado pelo Decreto Presidencial no 2.519 em 16 de março de 1998. $\mathrm{O}$ artigo $8 \mathrm{~h}$, que trata de espécies exóticas invasoras no corpo da $\mathrm{CDB}$, convoca os países signatários a "prevenir a introdução de, controlar ou erradicar espécies exóticas que ameacem ecossistemas, hábitats ou espécies"; além de encorajá-los a priorizar e implementar estratégias e planos de ação para espécies exóticas invasoras. Entretanto, antes que o Brasil pudesse atender a essas demandas, era necessário identificar quais espécies exóticas invasoras ocorriam no país e os locais e ambientes ameaçados ou já invadidos.

Reconhecendo a lacuna de conhecimento sobre invasões biológicas no Brasil, o Ministério do Meio Ambiente abriu, em 2004, com recursos do Banco Mundial, um edital para a elaboração de um Informe Nacional sobre Espécies Exóticas Invasoras (designado "Informe" deste ponto em diante). Os objetivos do Informe foram a coleta, a sistematização e a divulgação de informações sobre espécies exóticas invasoras no Brasil. O Informe foi elaborado nos anos de 2004 e 2005, tendo sido subdividido em cinco temas: espécies de ambientes terrestres, de águas continentais, de ambientes marinhos, que afetam áreas destinadas à produção e que afetam a saúde humana. Os relatórios trataram de espécies exóticas invasoras atuais e potenciais e incluíram uma avaliação sobre a estrutura existente no país para a prevenção e o controle de invasões biológicas. O foco deste artigo é exclusivamente em espécies exóticas invasoras em ambientes terrestres, 
embora inevitavelmente alguns pontos tratados também tenham relação com os outros temas. Em dezembro de 2005, com a finalização do Informe, foi realizado em Brasília o primeiro Simpósio Nacional sobre espécies exóticas invasoras, para o qual se inscreveram cerca de 700 pessoas. O Informe e o Simpósio Nacional são considerados um marco único, pois é impossível dissociar os efeitos dessas duas ações no contexto deste trabalho.

Em 2015 completam-se dez anos da realização do Informe Nacional sobre Espécies Exóticas Invasoras que afetam ambientes terrestres e do Simpósio Nacional. Neste trabalho, nossos objetivos são: (i) sistematizar e avaliar os avanços obtidos ao longo desse período nas esferas científica, legal e de gestão pública; (ii) identificar as principais lacunas de conhecimento, manejo e gestão de invasões biológicas; e (iii) indicar ações prioritárias para aumentar e melhorar o conhecimento sobre invasões biológicas e melhorar a gestão do tema no Brasil.

\section{Material e Métodos}

\section{Produção de conhecimento}

Para quantificar a produção científica ao longo dos anos, usamos a Web of Science, o Google Scholar e bibliotecas pessoais de artigos científicos dos autores. Os termos de busca foram invas*, Brazil ou Brasil. Incluímos todos os trabalhos encontrados em periódicos científicos que tratam de invasões biológicas ou espécies exóticas invasoras no Brasil, realizados ou focados em ambientes terrestres. Não incluímos trabalhos que apenas citam espécies exóticas invasoras ou invasões biológicas, mas cujo escopo não envolveu biologia de invasões, por exemplo, ao mencionar espécies exóticas invasoras apenas na introdução do manuscrito. Cada artigo foi classificado quanto ao ano de publicação, ao grupo taxonômico, à região geográfica, ao hábitat e à categoria de publicação (biologia, ecologia, genética, manejo, nota de ocorrência, revisão de literatura e sociobiologia). Os dados referentes ao Informe foram extraídos do Banco de Dados I3N Brasil de espécies exóticas invasoras, mantido pelo Instituto Hórus de Desenvolvimento e Conservação Ambiental (www.institutohorus.org.br), uma vez que o governo federal até hoje não publicou uma lista nacional oficial de espécies exóticas invasoras por meio de um instrumento normativo.

Analisamos os dados com modelos lineares generalizados (GLM) com distribuição de Poisson, usando o número de publicações como variável dependente e o grupo taxonômico, a região geográfica, o hábitat ou a categoria de publicação como variável independente. Os dados de 2015 foram excluídos da análise do número de publicações por ano em função de o ano estar incompleto. Realizamos um teste T unilateral para comparar o número de publicações por ano antes e depois da publicação do Informe.

\section{Marcos legais}

Uma lista de marcos legais municipais, estaduais e nacionais referentes a espécies exóticas invasoras no Brasil e/ou a medidas de prevenção, gestão e manejo de invasões biológicas está disponível no website do Instituto Hórus (INSTITUTO HÓRUS, 2015). A partir desta lista, caracterizamos cada marco legal em relação à esfera (federal, estadual e municipal), ao tipo e órgão responsável pela publicação, ao número e ano de publicação e ao tema especificamente tratado. Foram consideradas tanto normativas específicas sobre espécies exóticas invasoras quanto aquelas que tratam de políticas e questões ambientais mais amplas, porém fazem menção ao tema (por exemplo, Lei de Crimes Ambientais, Política Nacional de Biodiversidade, entre outras).

\section{Resultados}

\section{Produção de conhecimentos}

Encontramos 143 artigos científicos que tratam de invasões biológicas ou de espécies exóticas invasoras no Brasil realizados ou focados em ambientes terrestres (Anexo 1). Os primeiros artigos são de $1999(\mathrm{n}=3)$, sendo o número de publicações crescente a cada ano $(\mathrm{z}=7,36 ; \mathrm{p}<0,001 ;$ Figura 1$)$. A média de artigos publicados por ano após a realização do Informe é sete vezes maior do que antes de sua realização, em 2005 
FIGURA 1: Número de publicações científicas por ano feitas no Brasil ou sobre o Brasil cujo tema envolve biologia de invasões. A linha pontilhada vertical representa o ano do término no Informe Nacional Sobre Espécies Exóticas Invasoras e a linha sólida representa o ajuste do modelo linear generalizado (GLM). A linha laranja representa o crescimento anual do número de publicação antes do Informe (>2005) e a linha roxa representa o crescimento anual do número de publicação depois do Informe (2005-2014). A área sombreada representa o intervalo de confiança (95\%) do ajuste do GLM.

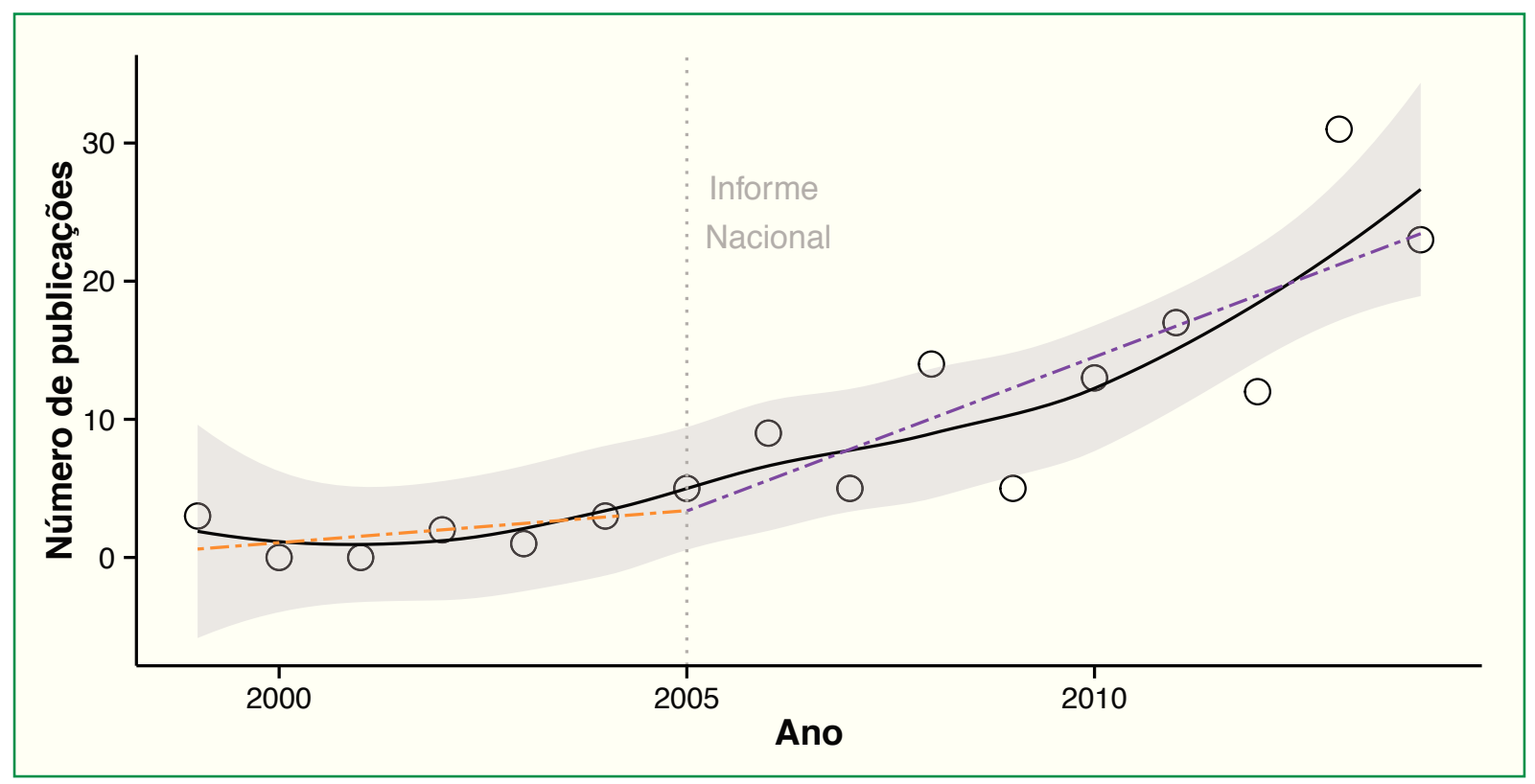

(antes $=2$ artigos.ano ${ }^{-1}$; depois $=14,3$ artigos. ano $^{-1}$; $\mathrm{t}=-4,26 ; \mathrm{df}=8,9 ; \mathrm{p}=0,001)$. Quase a metade dos estudos publicados tem foco na ecologia de invasões $(\mathrm{n}=70)$ e cerca de um quinto em aspectos biológicos de espécies exóticas invasoras $(n=26)$. Treze por cento dos artigos $(n=18)$ são revisões de literatura, $11 \%$ $(\mathrm{n}=16)$ são notas de ocorrência, $6 \%(\mathrm{n}=9)$ são estudos de manejo de invasões biológicas, $2 \%(n=3)$ são estudos genéticos e menos de $1 \%(n=1)$ tem foco em sociobiologia.

Plantas $(\mathrm{n}=72)$ foram mais estudadas do que animais $(n=49)$ e 22 trabalhos abordaram diversos grupos taxonômicos. Dentre as plantas, árvores $(\mathrm{n}=41 ; \mathrm{z}=4,2 ; \mathrm{p}<0,001)$ e gramíneas $(\mathrm{n}=18 ; \mathrm{z}=2,95$; $\mathrm{p}=0,003)$ foram desproporcionalmente mais estudadas do que palmeiras $(n=6)$, arbustos $(n=2)$, lianas $(n=1)$, pteridófitas $(n=1)$ e outras herbáceas $(n=3)$. Dentre os animais, insetos $(\mathrm{n}=14 ; \mathrm{z}=2,57 ; \mathrm{p}=0,01)$ e mamíferos $(\mathrm{n}=15 ; \mathrm{z}=2,67 ; \mathrm{p}=0,007)$ foram desproporcionalmente mais estudados do que moluscos $(n=7)$, répteis $(n=6)$, anfíbios $(\mathrm{n}=5)$ e anelídeos $(\mathrm{n}=2)$.
Praticamente um terço dos estudos $(n=44)$ foi realizado na região Sudeste do país, enquanto 27 estudos tiveram abrangência nacional; 25 referiam-se à região Sul, 19 à região Centro-Oeste, 17 à região Nordeste e apenas quatro à região Norte. Quatro publicações tiveram abrangência global e outras duas tiveram abrangência continental (América do Sul). Apenas uma publicação foi oriunda de estudos em ilhas oceânicas (Figura 2). Os hábitats mais estudados foram as Florestas Ombrófilas Densa e Mista $(\mathrm{n}=34)$, junto com estudos que incluíram múltiplos hábitats $(\mathrm{n}=35)$. Vinte estudos foram feitos no Cerrado, enquanto 15 focaram na Caatinga, 10 em ambiente urbano, sete em Formações Pioneiras de Origem Marinha (restingas), seis em Floresta Estacional, cinco em áreas rurais, quatro no Pampa, dois em ambiente agroflorestal, dois no Pantanal, um na Savana Amazônica, um em Floresta Amazônica e um foi realizado exclusivamente em laboratório.

\section{Marcos legais}

Encontramos 65 normativas publicadas no Brasil que fazem alguma referência a espécies exóticas 
FIGURA 2: Número de publicações científicas por ano feitas por regiões geográficas cujo tema envolve biologia de invasões.

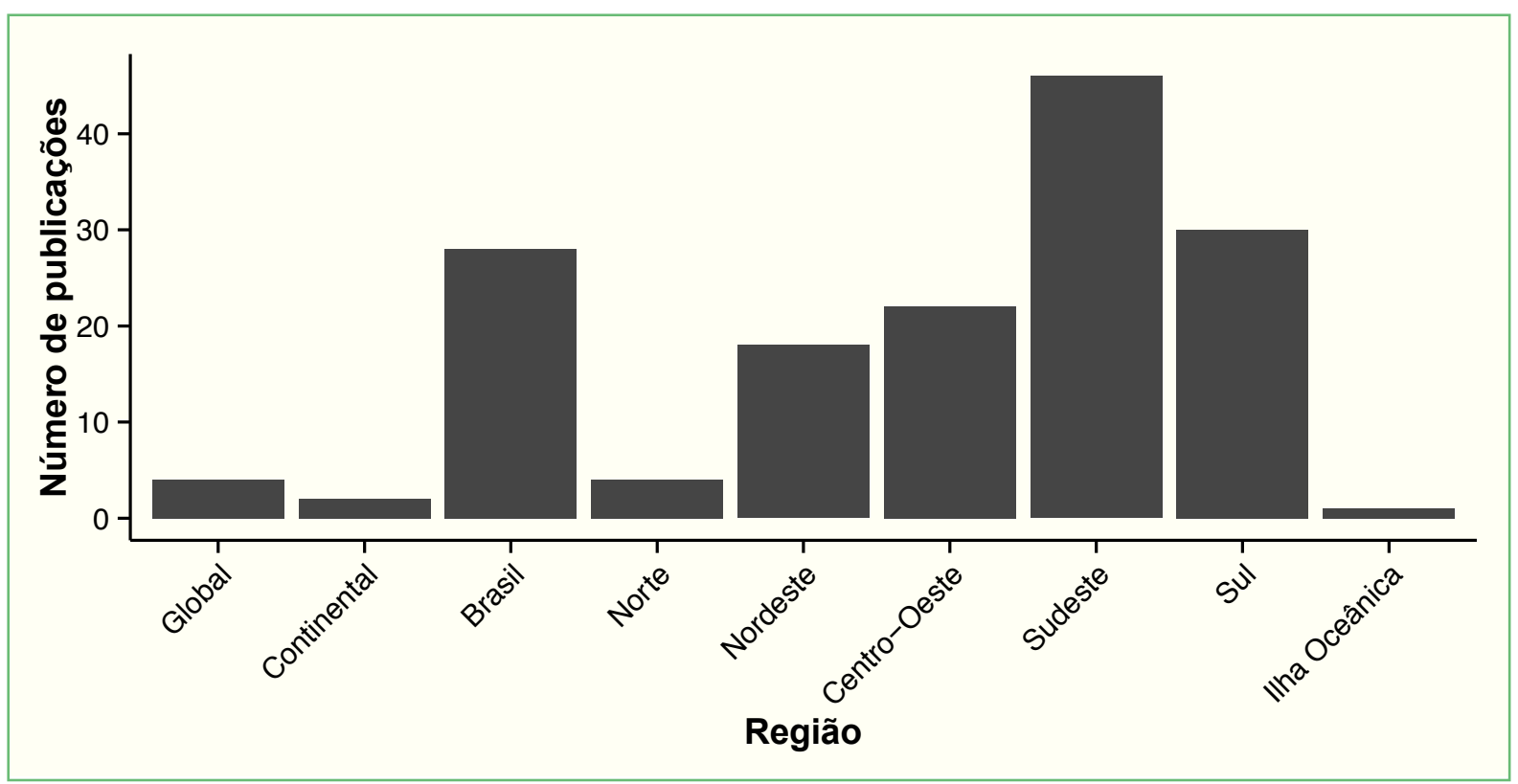

invasoras em ambientes naturais (Anexo 2). Cerca de $18,5 \%(\mathrm{n}=12)$ das normativas foi publicada entre 1993 e 2004 , enquanto $81,5 \%(n=53)$ entrou em vigor entre 2005 e 2014, após a realização do Informe (Figura 3). Vinte e quatro normativas (37\%) foram publicadas na esfera federal, $30(46 \%)$ na estadual e $11(17 \%)$ na municipal. A normativa mais antiga em termos federais é a Portaria n 205 do Ministério da Agricultura, de 13 de março de 1979, que proibiu o comércio, a importação, a exportação e o transporte de Eragrostis plana Nees (capim-annoni 2). A normativa mais antiga em nível estadual é uma Resolução publicada pelo Instituto Ambiental do Paraná em 1997, que define Tecoma stans (L.) Juss. ex Kunth (amarelinho) como planta nociva no estado do Paraná. Na esfera municipal todas as normativas são posteriores a 2005, sendo a normativa mais antiga de 2007, publicada no município de Bauru, São Paulo.

Quando consideradas somente as normativas específicas ao tema de invasões biológicas ou a espécies exóticas invasoras, o número de normativas federais passa a ser sete, o de estaduais passa a ser 26 e o número de normativas municipais não se altera $(n=11)$ - ou seja, todas as normativas municipais avaliadas são específicas. Até o presente momento nenhuma lei ou decreto federal específico sobre o tema foi publicado, sendo as normativas específicas uma Deliberação CONABIO (Comissão Nacional de Biodiversidade, responsável pela execução das diretrizes da $\mathrm{CDB}$ ) em 2006; três Instruções Normativas do IBAMA nos anos de 2005, 2012 e 2013; uma Resolução da CONABIO em 2009 (Estratégia Nacional para Espécies Exóticas Invasoras); e uma Portaria do IBAMA em 2010. Na esfera estadual, uma lei, dez instruções normativas, dez portarias, duas deliberações e três resoluções específicas foram publicadas nos estados da região Sul (Paraná, Santa Catarina e Rio Grande do Sul) e em dois estados da região Sudeste (São Paulo e Espírito Santo).

Listas oficiais de espécies exóticas invasoras foram publicadas nos estados de Paraná, Rio Grande do Sul, Santa Catarina e São Paulo por meio de portarias das agências ambientais estaduais (RS e PR) e de resoluções do Conselho Estadual de Meio Ambiente (CONSEMA) (SP e SC). A espécie E. plana foi objeto da única Lei publicada em âmbito estadual no Brasil, quando a Lei 13.187/2009 instituiu o Programa de controle de E. plana no estado do Rio Grande do Sul. Sus scrofa (Linnaeus 1758) (javali) e T. stans também foram alvo de normativas específicas no Paraná, Santa Catarina e Rio Grande do Sul. No Espírito Santo e no Paraná, 
FIGURA 3: Número acumulado de marcos legais existentes no Brasil sobre espécies exóticas invasoras por ano.

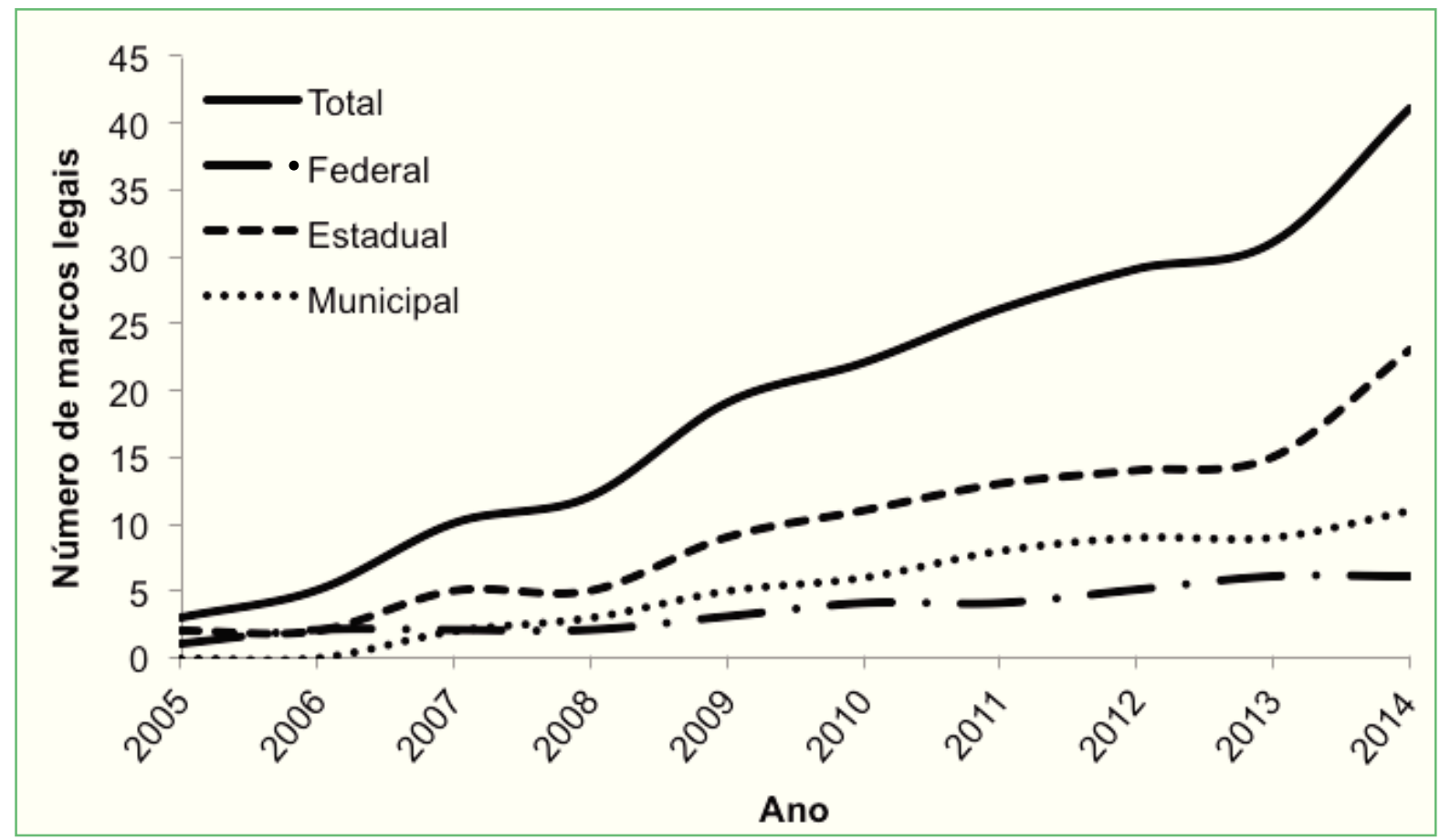

há normativas publicadas pelas agências ambientais estaduais referentes a programas de manejo de espécies exóticas invasoras em unidades de conservação; já em Santa Catarina, a Lei 14.675/09, que instituiu o Código Ambiental de Santa Catarina, determinou que a agência ambiental estadual seria responsável pela implantação de uma lista de referência e de um programa de controle de espécies exóticas invasoras em um prazo de quatro anos da data de publicação da referida lei.

$\mathrm{Na}$ esfera municipal, foram publicadas uma lei, um decreto, cinco resoluções e duas portarias entre 2007 e 2014 nos municípios do Rio de Janeiro (RJ), Bauru (SP), São Paulo (SP) e Curitiba (PR) (Anexo 2). Esses quatro municípios contam com listas oficiais de espécies exóticas invasoras, ainda que parciais. Destaca-se ainda que as demais normativas referem-se à instituição de políticas e programas municipais de controle e erradicação de espécies exóticas invasoras. Para Lissachatina fulica (Bowdich 1822) (caramujo-giganteafricano) foi publicada uma portaria que estabelece estratégias para implementação de plano de controle em Bauru. Em Florianópolis (SC), a Lei 9.097/2012 instituiu uma política municipal de remoção e a substituição de espécies dos gêneros Pinus L., Eucalyptus L'Hér. e Casuarina L. por espécies nativas, tarefa a ser realizada no período de dez anos.

\section{Discussão}

Globalmente, os primeiros estudos científicos em biologia de invasões datam do final da década de 1970 (RICHARDSON; PYŠEK, 2008). No Brasil, os primeiros trabalhos foram publicados em 1999. Enquanto que globalmente o ponto de inflexão do número de publicações em biologia de invasões ocorreu entre os anos 1995 e 2000 (RICHARDSON; PYŠEK, 2008), no Brasil este crescimento só foi atingido em 2008 (Figura 1). A proporção de aumento no número de estudos para o Brasil também é menor do que a observada globalmente. Embora seja impossível associar o crescimento da biologia de invasões no Brasil exclusivamente à realização do Informe e do Simpósio nacionais, é provável que estes tenham tido um papel fundamental nesse crescimento. Apesar dos resultados do Informe não terem sido integralmente publicados 
pelo Ministério do Meio Ambiente, havendo apenas um volume sobre espécies exóticas invasoras em ambientes marinhos, os levantamentos de dados envolveram profissionais de todo o Brasil e de diversas áreas, criando uma demanda por informações e fomentando discussões até então inexistentes sobre quais são e onde estão as espécies exóticas invasoras no Brasil. Além disso, por esforço e iniciativa do Instituto Hórus com apoios parciais da The Nature Conservancy (TNC), da área temática de espécies invasoras (I3N) da Rede InterAmericana de Informação sobre Biodiversidade (IABIN) e do Programa Global para Espécies Invasoras (GISP), os dados levantados no Informe foram disponibilizados ao público na internet e desde então as informações vêm sendo curadas e atualizadas pela equipe do Hórus. Os dados do Informe disponibilizados pelo banco de dados do Instituto Hórus já contribuíram com diversas publicações científicas (e.g., ZENNI; ZILLER, 2011; ZILLER; DECHOUM, 2013; SAMPAIO; SCHMIDT, 2013; ZENNI, 2014).

Os trabalhos publicados cobrem uma ampla gama de grupos biológicos, regiões geográficas (Figura 2) e hábitats. Assim como a maior parte das plantas exóticas invasoras no Brasil são árvores (ZENNI; ZILLER, 2011), a maioria dos estudos com plantas invasoras também são sobre árvores. Por outro lado, enquanto no Informe foram identificadas 31 espécies de plantas herbáceas exóticas invasoras, encontramos apenas três estudos publicados sobre esse grupo. Os esforços científicos têm sido mais equilibrados para a fauna, pois os números de animais exóticos invasores e números de publicações são equivalentes para os grupos biológicos, ainda que haja predomínio de trabalhos sobre mamíferos e insetos. Todas as regiões do Brasil têm sido estudadas e o país tem feito parte de importantes análises continentais e globais (e.g., SIMBERLOFF et al., 2010; KULL et al., 2011; WILSON et al., 2011). Da mesma forma, todos os grandes ecossistemas brasileiros têm sido estudados de acordo com os níveis de severidade de invasões biológicas observados (ZENNI; ZILLER, 2011; ZENNI; DECHOUM, 2013; ZENNI, 2014).

Uma observação importante é que o escopo das pesquisas sobre espécies exóticas invasoras no Brasil é limitado. De modo geral, as pesquisas buscam avaliar aspectos específicos relacionados a interações entre uma espécie invasora e uma determinada espécie, população ou comunidade nativa. Poucos trabalhos têm foco em aspectos amplos da biologia de invasões, de forma a contribuir com avanços para a disciplina. A maioria dos trabalhos trata de estudos de caso específicos com baixo poder de inferência ou generalização. Ainda, a quase totalidade dos trabalhos é de observação, priorizandose a detecção de padrões ao invés da determinação de mecanismos ecológicos e evolutivos. Outro ponto que chama a atenção é a elevada porcentagem $(12,6 \%)$ de revisões de literatura para o número de estudos primários. A proporção de uma revisão para cada oito artigos primários é elevada e indica que as revisões tendem a ser baseadas em poucos dados ou em dados oriundos de outras fontes que não publicações científicas. Notamos também que a pesquisa básica sobre invasões biológicas prepondera sobre a pesquisa em manejo e controle.

Ao menos uma iniciativa foi criada para melhorar o cenário da pesquisa em invasões biológicas. Em 2012 um grupo de pesquisadores propôs a criação de uma rede latino-americana de trabalho sobre plantas exóticas invasoras (Latin American Invasive Plants Network; GARDENER et al., 2012), com o objetivo de promover uma agenda de pesquisa que incorporasse lições aprendidas em regiões onde a biologia de invasões está mais desenvolvida. Entre 2011 e 2014 a Rede produziu cinco edições do seu Boletim, publicando um total de 27 artigos. Deste total, quatro artigos $(\sim 15 \%)$ têm autoria ou coautoria de brasileiros. Em 2014, a Rede criou a Bioinvasiones, uma revista científica sobre invasões biológicas na América Latina e Caribe, mas que até o final de 2015 não teve nenhum volume publicado (http:// bioinvasiones.org). Contudo, nenhum pesquisador ou pesquisadora brasileiros compõe o corpo editorial da Revista, que conta com editores sediados na Argentina, Chile, Estados Unidos, Nova Zelândia e Venezuela. Ainda assim, está claro que a ciência brasileira despertou para a biologia de invasões e que o número de estudos sobre o tema vem crescendo aceleradamente. Por outro lado, os dados também sugerem que a pesquisa brasileira está defasada nessa área em relação ao padrão internacional atual. 
Seguindo o mesmo padrão que o observado para o número de publicações, percebemos um aumento no número de normativas específicas sobre espécies exóticas invasoras nas três esferas governamentais após 2005, ano de conclusão do Informe. Em termos de visibilidade do tema no Brasil, apesar de o governo federal não haver, até o presente, publicado uma lista oficial ou sequer os resultados completos do Informe, a divulgação dos dados do Informe pelo terceiro setor (Instituto Hórus) chamou a atenção de técnicos e gestores públicos (MACHADO et al., 2008; PETENON; PIVELLO, 2008). Como consequência, o tema foi colocado em pauta em programas e projetos públicos, especialmente nas regiões Sul e Sudeste, evidenciando a necessidade de normativas específicas que viabilizassem a gestão do tema. Destaca-se, ainda, o quanto o tema foi permeando as esferas de gestão ao longo dos últimos dez anos, chegando mais recentemente à esfera municipal.

Espécies exóticas invasoras são legalmente reconhecidas como um problema ambiental no Brasil para o qual estratégias de manejo e gestão são necessárias. Apesar de existirem decretos e leis federais mais amplos que façam referência ao tema, tais como o Decreto 4.339/2002, que institui a Política Nacional de Biodiversidade, a Lei 9.605/1998 e o Decreto 3.179/1999, que respectivamente estabelece e regulamenta crimes ambientais no Brasil, nenhum instrumento legal de maior força, como um decreto ou uma lei, institui uma política nacional específica para o tema, ou sequer uma lista de espécies de referência para as diferentes regiões. Falta orientação política em nível nacional, especialmente porque após a publicação da Resolução CONABIO 05 de 2009 (Estratégia Nacional para Espécies Exóticas Invasoras) não houve iniciativa de implantação da estratégia nacional por parte do Ministério do Meio Ambiente. Por conta disso, alguns estados da Federação têm assumido, ao menos parcialmente, o compromisso pelo governo federal no que se refere às metas da $\mathrm{CDB}$.

As iniciativas estaduais são coerentes com a estratégia nacional e a CDB. Na falta de uma coordenação federal, essa consonância entre as iniciativas estaduais foi articulada pelo Instituto Hórus, que tem participado ativamente dos trabalhos em todos os estados. As normativas estaduais e municipais estão focadas na publicação de estratégias de gestão, de listas oficiais de referência e da publicação de normas complementares que visam estabelecer regras para o uso de espécies constantes nessas listas. No estado de São Paulo, por exemplo, constam na normativa publicada um pequeno número de espécies, em virtude de conflitos pela inclusão de espécies utilizadas em sistemas produtivos. Os estados do Paraná e de Santa Catarina publicaram listas extensas em 2007 e 2012, respectivamente. A Secretaria Estadual de Meio Ambiente do Rio Grande do Sul publicou, em 2013, uma lista extensa de espécies e, em 2014, diversas instruções normativas regulamentando o uso destas, tais como Acacia mearnsii De Wild. (acácianegra), Pinus spp., Urochloa spp. P. Beauv. (braquiárias), Archontophoenix cunninghamiana $\mathrm{H}$. Wendl. \& Drude (palmeira-imperial) e Lithobates catesbeianus (Shaw 1802) (rã-touro). As normativas regulamentam atividades que podem gerar invasões biológicas, como a criação ou o cultivo de espécies exóticas invasoras, e estabelecem limites para o uso dessas espécies. As listas publicadas têm por objetivo servir como referência técnica e não proibir o uso de espécies em geral, permitindo o uso produtivo, porém resguardando ambientes naturais de processos de invasão.

Invasões biológicas fornecem oportunidades únicas para a compreensão dos efeitos da introdução de espécies em comunidades ou ecossistemas, da força da adaptação local e de plasticidade fenotípica e das respostas dos organismos a condições ambientais (e.g., WARDLE et al., 2011; CAPLAT et al., 2013; MORAN; ALEXANDER, 2014). Contudo, invasões biológicas também são uma das principais causas de impactos ambientais globais e seu controle e manejo são fundamentais para a conservação da biodiversidade e de serviços ecossistêmicos (SIMBERLOFF et al., 2013). Neste sentido, a pesquisa deve caminhar lado a lado com as necessidades práticas de prevenção, detecção precoce, controle, manejo e apoio à gestão pública. O que se observou no Brasil nestes últimos dez anos, contudo, foi que pesquisadores focaram predominantemente em aspectos básicos da biologia de invasões sem se preocupar com o potencial desse conhecimento para a gestão do tema, enquanto gestores realizaram ações práticas e desenvolveram marcos legais com visão 
aplicada desvinculada do conhecimento científico produzido. Uma melhor integração dos aspectos teóricos e aplicados de invasões biológicas como apoio à gestão pública é altamente desejável e depende da inclusão do tema em mais cursos profissionais, de mais oportunidades para estudos de pós-graduação na área contando com interações com profissionais e pesquisadores de outros países e também da inclusão da problemática em níveis de ensino básico e médio.

Nos últimos dez anos, invasões biológicas receberam mais atenção no Brasil do que em qualquer outro período. Embora o tema tenha se tornado relevante no país somente 20 anos depois do surgimento da biologia de invasões, o crescimento no número de pesquisas sobre invasões biológicas e a publicação de um arcabouço legal vêm rapidamente preenchendo a lacuna existente. Mesmo não sendo possível atribuir este crescimento exclusivamente à realização do Informe e do Simpósio Nacional em 2005, é bastante provável que ambos tenham tido um papel fundamental no início desse processo. Infelizmente, o Ministério do Meio Ambiente não tem se dedicado a implantar as ações definidas na Estratégia Nacional para Espécies Exóticas Invasoras publicada em 2009, tampouco a meta 9 de Aichi estabelecida no âmbito da CDB.

O tema de invasões biológicas precisa estar amplamente difundido na sociedade para que ela possa contribuir regional e internacionalmente com conhecimento, com a consolidação de ações concretas em diferentes partes do país e, finalmente, com a mitigação de riscos ambientais, econômicos e sociais de invasões biológicas. Atualmente, o protagonismo da geração de conhecimento no Brasil está concentrado em poucos grupos de pesquisa vinculados a universidades, enquanto o viés prático está com o terceiro setor e em implementação por parte de alguns estados e municípios. Nos próximos anos, projetos de pesquisa, manejo e gestão devem ser mais ambiciosos e relevantes para avançar o tema no país, contribuir para seu avanço em termos internacionais, embasar ações práticas de manejo e consolidar um arcabouço legal integrado que efetivamente permita prevenir, manejar e controlar invasões biológicas.

\section{Agradecimentos}

Ao CNPq, pela concessão da bolsa ao primeiro autor (processo 313926/2014-0). À CAPES, pela concessão da bolsa ao segundo autor (projeto no A114/2013). A André F. Mendonça, que auxiliou na busca de referências bibliográficas.

\section{Referências}

CAPLAT, P.; CHEPTOU, P. O.; DIEZ, J.; GUISAN,A.; LARSON, B. M. H.; MACDOUGALL, A. S.; PELTZER, D. A.; RICHARDSON, D. M.; SHEA, K.; VAN KLEUNEN, M.; ZHANG, R.; BUCKLEY, Y. M. Movement, impacts and management of plant distributions in response to climate change: insights from invasions. Oikos, Copenhagen, v. 122, n. 9, p. 1265-1274, 2013.

DIDHAM, R. K.; TYLIANAKIS, J. M.; GEMMELL, N. J.; RAND, T. A.; EWERS, R. M. The interactive effects of habitat loss and species invasion on native species decline. Trends in Ecology \& Evolution, Cambridge, v. 22, n. 9, p. 489-496, 2007.

DRAKE, J. A.; MOONEY, H. A.; DI CASTRI, F.; GROVES, R. H.; KRUGER, F. J.; REJMÁNEK, M.; WILLIAMSON, M. Biological invasions: a global perspective. Chichester: John Wiley \& Sons, 1989. $525 \mathrm{p}$.

ELTON, C. S. The ecology of invasions by animals and plants. Chicago: University of Chicago Press, 1958. 116 p.

GARDENER, M. R.; BUSTAMANTE, R. O.; HERRERA, I.; DURIGAN, G.; PIVELLO, V. R.; MORO, M. F.; STOLL, A.; LANGDON, B.; BARUCHI, Z.; RICO, A.; ARREDONDONUNEZ, A.; FLORES, S. Plant invasions research in Latin America: fast track to a more focused agenda. Plant Ecology \& Diversity, Abingdon, v. 5, n. 2, p. 225-232, 2012.

GILBERT, B.; LEVINE, J. M. Plant invasions and extinction debts. Proceedings of the National Academy of Sciences of the United States of America, Washington, v. 110, n. 5, p. 1744-1749, 2013.

INSTITUTO HÓRUS. Marcos legais. 2015. Disponível em: $<$ http://www.institutohorus.org.br/index.php? modulo=marcos legais>. Acesso em: 8 jul. 2015.

KATSANEVAKIS, S.; WALLENTINUS, I.; ZENETOS, A.; LEPPÄKOSKI, E.; ÇINAR, M. E.; OZTÜRK, B.; GRABOWSKI, M.; GOLANI, D.; CARDOSO, A. C. Impacts of invasive alien marine species on ecosystem services and biodiversity: a panEuropean review. Aquatic Invasions, Helsinki, v. 9, n. 4, p. 391423, 2014.

KULL, C. A.; SHACKLETON, C. M.; CUNNINGHAM, P. J.; DUCATILLON, C.; DUFOUR-DROR, J. -M.; ESLER, K. J.; FRIDAY, J. B.; GOUVEIA, A. C.; GRIFFIN, A. R.; MARCHANTE, E.; MIDGLEY, S. J.; PAUCHARD, A.; RANGAN, H.; RICHARDSON, D. M.; RINAUDO, T.; TASSIN, J.; URGENSON, L. S.; VON MALTITZ, G. P.; ZENNI, R. D.; ZYLSTRA, M. J. Adoption, use and perception of Australian acacias around the world. Diversity and Distributions, Malden, v. 17, n. 5, p. 822$836,2011$. 
MACHADO, C. J. S.; OLIVEIRA, A. E. S.; MATOS, D. M. S.; PIVELLO, V. R; CHAME, M.; SOUZA, R. C. C. L.; CALAZANS, S. H.; SILVA, E. P. Recomendações para elaboração e consolidação de uma estratégia nacional de prevenção e controle das espécies exóticas no Brasil. Ciência e Cultura, São Paulo, v. 61, n. 1, p. 42-45, 2009.

MORAN, E. V.; ALEXANDER, J. M. Evolutionary responses to global change: lessons from invasive species. Ecology Letters, Montpellier, v. 17, n. 5, p. 637-649, 2014.

PETENON, D.; PIVELLO, V. R. Plantas invasoras: representatividade da pesquisa dos países tropicais no contexto mundial. Natureza \& Conservação, Curitiba, v. 6, n. 1, p. 65-77, 2008.

REASER, J. K.; MEYERSON, L. A.; CRONK, Q.; DE POORTER, M.; ELDREGE, L.G.; GREEN, E; KAIRO, M.; LATASI, P.; MACK, R. N.; MAUREMOOTOO, J.; O'DOWD, D.; ORAPA, W.; SASTROUTOMO, S.; SAUNDERS,A.; SHINE, C.; THRAINSSON, S.; VAIUTU, L. Ecological and socioeconomic impacts of invasive alien species in island ecosystems. Environmental Conservation, Cambridge, v. 34, n. 2, p. 1-14, 2007.

RICHARDSON, D. M.; PYŠEK, P. Fifty years of invasion ecology - the legacy of Charles Elton. Diversity and Distributions, Malden, v. 14, n. 2, p. 161-168, 2008.

SAMPAIO, A. B.; SCHMIDT, I. B. Espécies exóticas invasoras em unidades de conservação federais do Brasil. Biodiversidade Brasileira, Brasília, v. 3, n. 2, p. 32-49, 2013.

SIMBERLOFF, D.; MARTIN, J. -L.; GENOVESI, P.; MARIS, V.; WARDLE, D. A.; ARONSON, J.; COURCHAMP, F.; GALIL, B.; GARCÍA-BERTHOU, E.; PASCAL, M.; PYŠEK, P.; SOUSA, R.; TABACCHI, E.; VILÀ, M. Impacts of biological invasions: what's what and the way forward. Trends in Ecology \& Evolution, Cambridge, v. 28, n. 1, p. 58-66, 2013.

SIMBERLOFF, D.; NUÑEZ, M. A.; LEDGARD, N. J.; PAUCHARD, A.; RICHARDSON, D. M.; SARASOLA, M.; VAN WILGEN, B. W.; ZALBA, S. M.; ZENNI, R. D.; BUSTAMANTE, R.; PEÑA, E.; ZILLER, S. R. Spread and impact of introduced conifers in South America: lessons from other southern hemisphere regions. Austral Ecology, Alice Springs, v. 35, n. 5, p. 489-504, 2010.

VILÀ, M.; ESPINAR, J. L.; HEJDA, M.; HULME, P. E.; JAROŠÍK, V.; MARON, J. L.; PERGL, J.; SCHAFFNER, U.; SUN, Y.; PYŠEK, P. Ecological impacts of invasive alien plants: a metaanalysis of their effects on species, communities and ecosystems. Ecology Letters, Montpellier, v. 14, n. 7, p. 702-708, 2011.
WARDLE, D. A.; BARDGETT, R. D.; CALLAWAY, R. M.; VAN DER PUTTEN, W. H. Terrestrial ecosystem responses to species gains and losses. Science, New York, v. 332, n. 6035, p. 1273-1277, 2011.

WILSON, J. R. U.; GAIRIFO, C.; GIBSON, M. R.; ARIANOUTSOU, M.; BAKAR, B. B.; BARET, S.; CELESTIGRAPOW, L.; DITOMASO, J. M.; DUFOUR-DROR, J.-M.; KUEFFER, C.; KULL, C. A.; HOFFMANN, J. H.; IMPSON, F. A. C.; LOOPE, L. L.; MARCHANTE, E.; MARCHANTE, H.; MOORE, J. L.; MURPHY, D. J.; TASSIN, J.; WITT, A.; ZENNI, R. D.; RICHARDSON, D. M. Risk assessment, eradication, and biological control: global efforts to limit Australian acacia invasions. Diversity and Distributions, Malden, v. 17, n. 5, p. 1030-1046, 2011.

WINTER, M.; SCHWEIGER, O.; KLOTZ, S.; NENTWIG, W.; ANDRIOPOULOS P.; ARIANOUTSOU, M.; BASNOU, C.; DELIPETROU, P.; DIDŽIULIS, V.; HEJDA, M.; HULME, P. E.; LAMBDON, P. W.; PERGL, J.; PYŠEK, P.; ROYL, D. B.; KÜHN, I. Plant extinctions and introductions lead to phylogenetic and taxonomic homogenization of the European flora. Proceedings of the National Academy of Sciences of the United States of America, Washington, v. 106, n. 51, p. 21721-21725, 2009.

ZENNI, R. D. Analysis of introduction history of invasive plants in Brazil reveals patterns of association between biogeographical origin and reason for introduction. Austral Ecology, Alice Springs, v. 39, n. 4, p. 401-407, 2014.

ZENNI, R. D.; DECHOUM, M. S. Paisagens antropizadas e invasão por plantas exóticas. In: PERES, C. A.; BARLOW, J.; GARDNER, T. A.; VIEIRA, I. C. G. (Ed.). Conservação da biodiversidade em paisagens antropizadas do Brasil. Curitiba: Editora UFPR, 2013. p. 549-563.

ZENNI, R. D.; ZILLER, S. R. An overview of invasive plants in Brazil. Brazilian Journal of Botany, São Paulo, v. 34, n. 3, p. 431446, 2011.

ZILLER, S. R.; DECHOUM, M. S. Plantas e vertebrados exóticos invasores em unidades de conservação no Brasil. Biodiversidade Brasileira, Brasília, v. 3, n. 2, p. 25-31, 2013. 


\section{ANEXO 1}

Relação das 143 publicações científicas feitas no Brasil ou que incluem o Brasil sobre biologia de invasões usadas no artigo "Dez anos do Informe brasileiro sobre espécies exóticas invasoras: avanços, lacunas e direções futuras".

1. Abreu, R. C. R. \& G. Durigan. 2011. Changes in the plant community of a Brazilian grassland savannah after 22 years of invasion by Pinus elliottii Engelm. Plant Ecology \& Diversity 4(2-3):269-278.

2. Abreu, R. C. R. \& P. J. F. P. Rodrigues. 2010. Exotic tree Artocarpus heterophyllus (Moraceae) invades the Brazilian Atlantic Rainforest. Rodriguésia 61(4):677688.

3. Abreu, R. C. R.; F. F. M. Santos \& G. Durigan. 2014. Changes in plant community of Seasonally Semideciduous Forest after invasion by Schizolobium parahyba at southeastern Brazil. Acta Oecologica 54(57-64.

4. Aguiar Jr., A.; R. I. Barbosa; J. B. Barbosa \& M. Mourão Jr. 2014. Invasion of Acacia mangium in Amazonian savannas following planting for forestry. Plant Ecology \& Diversity 7(1-2):359-369.

5. Andrade, L. A.; JR. Fabricante \& A. S. Alves. 2008. The algarrobe Prosopis juliflora (Sw) DC.: impacts over phytodiversity and colonization strategies in invaded areas in Paraíba, Brazil. Nat Conservacao 6(2):169-175.

6. Andrade, L. A.; JR. Fabricante \& F. X. Oliveira. 2010. Impactos da invasão de Prosopis juliflora (sw.) DC.(Fabaceae) sobre o estrato arbustivo-arbóreo em áreas de Caatinga no Estado da Paraíba, Brasil. Acta Scientiarum 32(3):249-255.

7. Andrade, L. A.; JR. Fabricante \& F. X. Oliveira. 2009. Invasão biológica por Prosopis juliflora (Sw.) DC.: impactos sobre a diversidade e a estrutura do componente arbustivo-arbóreo da caatinga no estado do Rio Grande do Norte, Brasil. Acta Botânica Brasílica 23(4):935-943.

8. Anjos, L. A. \& C. F. D. Rocha. 2008a. The Hemidactylus mabouia Moreau de Jonnes, 1818 (Gekkonidae) lizard: an invasive alien species broadly distributed in Brazil. Nat Conservacao 6(1):196-207.

9. Anjos, L. A. \& C. F. D. Rocha. 2008b. Reproductive ecology of the invader species gekkonid lizard Hemidactylus mabouia in an area of southeastern Brazil. Iheringia. Série Zoologia 98(2):205-209.

10. Attias, N.; M. F. Siqueira \& H. G. Bergallo. 2013. Acácias australianas no Brasil: histórico, formas de uso e potencial de invasão. Biodiversidade Brasileira 3(2):74-96.

11. Auricchio, P. \& F. Olmos. 1999. Northward range extension for the european hare (Lepus europaeus pallas)
1778 (Lagomorpha - Leporidae) in Brazil. Publicações Avulsas do Instituto Pau Brasil 2(1-5.

12. Azevêdo-Gonçalves, C. F. \& C. N. Gonçalves. 2013. Registro da ocorrência de Hypochaeris chillensis (Asteraceae) e uma análise do potencial invasivo da espécie na Chapada Diamantina, Bahia. Biodiversidade Brasileira 3(2): 106-117.

13. Bao, F.; A. Pott; F. A. Ferreira \& R. Arruda. 2014. Soil seed bank of floodable native and cultivated grassland in the Pantanal wetland: effects of flood gradient, season and species invasion. Brazilian Journal of Botany 37(3):239-250.

14. Barbosa, E. G.; V. R. Pivello \& S. T. Meirelles. 2008. Allelopathic evidence in Brachiaria decumbens and its potential to invade the Brazilian cerrados. Brazilian Archives of Biology and Technology 51(4):825-831.

15. Barbosa, F. G.; V. D. Pillar; A. R. Palmer \& A. S. Melo. 2013. Predicting the current distribution and potential spread of the exotic grass Eragrostis plana Nees in South America and identifying a bioclimatic niche shift during invasion. Austral Ecology 38(3):260-267.

16. Barbosa, N. P. U.; G. W. Fernandes; M. A. A. Carneiro \& L. A. C. Junior. 2010. Distribution of alien invasive species and soil properties in proximity to paved roads and unpaved roads in a quartzitic mountainous grassland of southeastern Brazil (rupestrian fields). Biological Invasions 12(11):3745-3755.

17. Bastos, E. G. D.; A. F. B. de Araújo \& H. R. da Silva. 2005. Records of the rattlesnakes Crotalus durissus terrificus (Laurenti) (Serpentes,Viperidae) in the State of Rio de Janeiro, Brazil: a possible case of invasion facilitated by deforestation. Revista Brasileira De Zoologia 22(3):812-815.

18. Biondi, D. \& E. Muller. 2013. Invasive tree species in urban parks landscaping of Curitiba, PR. Floresta 43(1):69-82.

19. Bisaggio, E. L.; S. L. Alves; C. C. Santos Júnior \& C. H. B. Rocha. 2013. Búfalos ferais (Bubalus bubalis) em áreas protegidas: um estudo de caso na Reserva Biológica do Guaporé, RO. Biodiversidade Brasileira 3(2):243-260.

20. Blum, C. T.; M. Borgo \& A. C. F. Sampaio. 2008. Espécies exóticas invasoras na arborização de vias públicas de Maringá-PR. Revista da Sociedade Brasileira de Arborização Urbana 3(2):78-97. 
21. Bovendorp, R. S.; A. D. Alvarez \& M. Galetti. 2008. Density of the tegu lizard (Tupinambis merianae) and its role as nest predator at Anchieta Island, Brazil. Neotropical Biology and Conservation 3(1):9-12.

22. Bovendorp, R. S. \& M. Galetti. 2007. Density and population size of mammals introduced on a land-bridge island in southeastern Brazil. Biological Invasions 9(3):353-357.

23. Braga, E. P.; R. D. Zenni \& J. D. Hay. 2014. A new invasive species in South America: Pinus oocarpa Schiede ex Schltdl. BioInvasion Records 3(3):207-211.

24. Braks, M. A. H.; N. A. Honório; L. P. Lounibos; R. Lourenço-de-Oliveira \& SA Juliano. 2004. Interspecific competition between two invasive species of container mosquitoes, Aedes aegypti and Aedes albopictus (Diptera: Culicidae), in Brazil. Annals of the Entomological Society of America 97(1):130-139.

25. Brown, G. G.; S. W. James; A. Pasini; D. H. Nunes; N. P. Benito et al. 2006. Exotic, peregrine, and invasive earthworms in Brazil: Diversity, distribution, and effects on soils and plants. Caribbean Journal of Science 42(3):339-358.

26. Burgueño, L. E. T; M. S. Quadro; A. A. Barcelos; PdÁ Saldo; FdS Weber et al. 2013. Impactos ambientais de plantios de Pinus sp. em zonas úmidas: o caso do Parque Nacional da Lagoa do Peixe, RS, Brasil. Biodiversidade Brasileira 3(2):159-174.

27. Carvalho, F. A.; R. C. R. Abreu; K. A. R. T. Barros; S. N. Fonseca; D. S. Santiago et al. 2014a. The regenerating tree community in a 'novel ecosystem' dominated by the invasive species Pinus elliottii Engelm. Interciencia 39(5):307-312.

28. Carvalho, J.; A. M. Ferreira; M. Belão \& R. Boçon. 2014b. Exóticas invasoras nas rodovias BR 277, PR 508, PR 407, Paraná, Brasil. Floresta 44(2):249-258.

29. Cavalcante, A. \& I. Major. 2006. Invasion of alien plants in the Caatinga biome. Ambio 35(3):141-143.

30. Chapla, T. E. \& J. B. Campos. 2010. Allelopathic Evidence in Exotic Guava (Psidium guajava L.). Brazilian Archives of Biology and Technology 53(6):1359-1362.

31. Christianini, A. V. 2006. Fecundidade, dispersão e predação de sementes de Archontophoenix cunninghamiana H. Wendl. \& Drude, uma palmeira invasora da Mata Atlântica. Revista Brasileira de Botânica 29(4):587-594.

32. Costa, J. N. M. N. \& G. Durigan. 2010. Leucaena leucocephala (Lam.) de Wit (Fabaceae): invasive or ruderal? Revista Árvore 34(5):825-833.

33. Costa, M. D. \& F. A. B. Fernandes. 2010. First report of Lepus europaeus Pallas, 1778 (Mammalia, Lagomorpha,
Leporidae) in the south of the state of Minas Gerais and a synthesis of records known for the southeast of Brazil. Revista Brasileira de Zoociências 12(3):311-314.

34. Culik, M. P.; D. D. Martins; J. S. Zanuncio; M. J. Fornazier; J. A. Ventura et al. 2013. The invasive hibiscus mealybug Maconellicoccus hirsutus (Hemiptera: Pseudococcidae) and its recent range expansion in Brazil. Florida Entomologist 96(2):638-640.

35. Culik, M. P.; D. S. Martins; J. A. Ventura \& V. A. Costa. 2014. The invasive gall wasp Quadrastichus erythrinae (Hymenoptera: Eulophidae) in South America: is classical biological control needed? Biocontrol Science and Technology 24(8):971-975.

36. Dechoum, M. S. \& S. R. Ziller. 2012. Métodos para controle de plantas exóticas invasoras. Biotemas 26: 6977.

37. Depra, M.; J. L. Poppe; H. J. Schmitz; D. C. De Toni \& V. L. S. Valente. 2014. The first records of the invasive pest Drosophila suzukii in the South American continent. Journal of Pest Science 87(3):379-383.

38. Desbiez, A. L. J.; A Keuroghlian; U. Piovezan \& R. E. Bodmer. 2011. Invasive species and bushmeat hunting contributing to wildlife conservation: the case of feral pigs in a Neotropical wetland. Oryx 45(1):78-83.

39. Diamantidis, A. D.; JR. Carey; C. T. Nakas \& N. T. Papadopoulos. 2011. Population-specific demography and invasion potential in medfly. Ecology and Evolution 1(4):

40. Dias, J.; MAMA da Fonte; R. Baptista; MC Mantoani; DR Holdefer et al. 2013. Invasive alien plants in brazil: a nonrestrictive revision of academic works. Nat Conservacao 11(1):31-35.

41. Dislich, R.; N. Kisser \& V. R. Pivello. 2002. The invasion of a forest fragment in São Paulo (SP) by the Australian palm Archontophoenix cunninghamiana $\mathrm{H}$. Wendl. and Drude. Brazilian Journal of Botany 25(1):55-64.

42. Durigan, G.; N. M. Ivanauskas; M. J. B. Zakia \& R. C. R. de Abreu. 2013. Control of invasive plants: ecological and socioeconomic criteria for the decision making process. Nat Conservacao 11(1):23-30.

43. Emer, C. \& C. R. Fonseca. 2011. Araucaria Forest conservation: mechanisms providing resistance to invasion by exotic timber trees. Biological Invasions 13(1):189-202.

44. Espíndola, M. B.; F. C. Bechara; M. S. Bazzo \& A. Reis. 2005. Recuperação ambiental e contaminação biológica: aspectos ecológicos e legais. Biotemas 18(1):27-38.

45. Fabricante, JR.; K. C. T. de Araujo; L. A. de Andrade \& J. V. A. Ferreira. 2012. Biological invasion of Artocarpus heterophyllus Lam. (Moraceae) in an Atlantic Forest fragment in Northeastern Brazil: impacts 
on phytodiversity and soils of invaded sites. Acta Botanica Brasilica 26(2):399-407.

46. Fabricante, JR.; M. N. A. Oliveira \& J. A. Siqueira Filho. 2013. Aspectos da ecologia de Calotropis procera (Apocynaceae) em uma área de Caatinga alterada pelas obras do Projeto de Integração do Rio São Francisco em Mauriti, CE. Rodriguésia 64(3):647-654.

47. Falleiros, R. M.; R. D. Zenni \& S. R. Ziller. 2011. Invasão e manejo de Pinus taeda em campos de altitude do Parque Estadual do Pico Paraná, Paraná, Brasil. Floresta 41(1):123-134.

48. Ferreira, M. T. S. \& P. M. Maia-Barbosa. 2013. O fogo como facilitador da invasão biológica por Megathyrsus maximus (Poaceae: Panicoideae) na Terra Indígena Maxakali (MG): propostas para um manejo agroecológico integrado e adaptativo. Biodiversidade Brasileira 3(2):159-174.

49. Fischer, F. M.; J. M. Oliveira; A. L. P. Dresseno \& V. D. Pillar. 2014. The role of invasive pine on changes of plant composition and functional traits in a coastal dune ecosystem. Nat Conservacao 12(1):19-23.

50. Fischer, M. L. 2009. Reactions of the invasive alien species Achatina fulica to abiotic factors: perspectives for the management. Zoologia 26(3):379-385.

51. Fischer, M. L. \& E. Colley. 2005. Espécie invasora em reservas naturais: caracterização da população de Achatina fulica Bowdich, 1822 (mollusca - achatinidae) na Ilha Rasa, Guaraqueçaba, Paraná, Brasil. Biota Neotropica 5(1):127-144.

52. Fischer, M. L.; M. Simião; E. Colley; R. D. Zenni; D. A. T. Silva et al. 2006. O caramujo exótico invasor na vegetação nativa em Morretes, PR: diagnóstico da população de Achatina fulica Bowdich, $1822 \mathrm{em}$ um fragmento de Floresta Ombrófila Densa Aluvial. Biota Neotropica 6(2):1-5.

53. Focht, T. \& R. B. de Medeiros. 2012. Prevention of natural grassland invasion by Eragrostis plana Nees using ecological management practices. Rev Bras Zootecn 41(8): 1816-1823.

54. Fonseca, C. R; D. L Guadagnin; C. Emer; S. Masciadri; P. Germain et al. 2013. Invasive alien plants in the Pampas grasslands: a tri-national cooperation challenge. Biological Invasions 15(8):1751-1763.

55. Fonseca, R. L.; P. R. Guimaraes; S. R. Morbiolo; R. Scachetti-Pereira \& A. T. Peterson. 2006. Predicting invasive potential of smooth crotalaria (Crotalaria pallida) in Brazilian national parks based on African records. Weed Science 54(3):458-463.

56. Frigeri, E.; C. R. Cassano \& R. Pardini. 2014. Domestic dog invasion in an agroforestry mosaic in southern
Bahia, Brazil. Tropical Conservation Science 7(3):508528.

57. Galego, L. G. C. \& C. M. A. Carareto. 2010. Scenario of the spread of the invasive species Zaprionus indianus Gupta, 1970 (Diptera, Drosophilidae) in Brazil. Genetics and molecular biology 33(4):767-773.

58. Gardener, M. R.; R. O. Bustamante; I. Herrera; G. Durigan; V. R. Pivello et al. 2012. Plant invasions research in Latin America: fast track to a more focused agenda. Plant Ecology \& Diversity 5(2):225-232.

59. Giovanelli, J. G. R.; C. F. B. Haddad \& J. Alexandrino. 2008. Predicting the potential distribution of the alien invasive American bullfrog (Lithobates catesbeianus) in Brazil. Biological Invasions 10(5):585-590.

60. Gomes, F. B. R. \& R. C. Lima-Gomes. 2011. Registro ocasional da predação da pomba-de-bando (Zenaida auriculata des Murs, 1847) pelo sagüi-do-cerrado (Callithrix penicillata E. Geoffroy, 1812) no interior de São Paulo, SP. Neotropical Primates 18(2):68-70.

61. Gomes, P. \& M. Sobral-Leite. 2013. Crystalline rock outcrops in the Atlantic Forest of northeastern Brazil: vascular flora, biological spectrum, and invasive species. Brazilian Journal of Botany 36(2):111-123.

62. Gomes, SR; JB Picanco; E Colley; AI Agudo-Padron; E Nakano et al. 2011. Newly introduced and invasive land slug in Brazil: Meghimatium pictum (Gastropoda, Philomycidae) from China. Proceedings of the Academy of Natural Sciences of Philadelphia 161(87-95.

63. Hendges, C.; V. Fortes \& M. S. Dechoum. 2012. Consumption of the invasive alien species Hovenia dulcis Thumb.(Rhamnaceae) by Sapajus nigritus Kerr, 1792 in a protected area in southern Brazil. Revista Brasileira de Zoociências 14(1):255-260.

64. Hoffmann, W. A. \& M. Haridasan. 2008. The invasive grass, Melinis minutiflora, inhibits tree regeneration in a Neotropical savanna. Austral Ecology 33(1):29-36.

65. Hoffmann, W. A.; V Lucatelli; F. J. Silva; I. N. C. Azeuedo; M. D. Marinho et al. 2004. Impact of the invasive alien grass Melinis minutiflora at the savannaforest ecotone in the Brazilian Cerrado. Diversity and Distributions 10(2):99-103.

66. Horowitz, C.; C. R. Martins \& B. M. T. Walter. 2013a. Flora exótica no Parque Nacional de Brasília: levantamento e classificação das espécies. Biodiversidade Brasileira 3(2):50-73.

67. Horowitz, C.; A. S. Oliveira; V. Silva; G. Pacheco \& R. I. Sobrinho. 2013b. Manejo da flora exótica invasora no Parque Nacional de Brasília: contexto histórico e atual. Biodiversidade Brasileira 3(2):217-236. 
68. Kaefer, I. L.; R. A. Boelter \& S. Z. Cechin. 2007. Reproductive biology of the invasive bullfrog Lithobates catesbeianus in southern Brazil. Annales Zoologici Fennici 44(6):435-444.

69. Koehler-Santos, P.; A. P. Lorenz-Lemke; V. C. Muschner; S. L. Bonatto; F. M. Salzano et al. 2006. Molecular genetic variation in Passiflora alata (Passifloraceae), an invasive species in southern Brazil. Biological Journal of the Linnean Society 88(4):611-630.

70. Kull, C. A.; C. M. Shackleton; P. J. Cunningham; C. Ducatillon; J. M. Dufour-Dror et al. 2011. Adoption, use and perception of Australian acacias around the world. Diversity and Distributions 17(5):822-836.

71. Leal, L. C.; M. V. Meiado; A. V. Lopes \& I. R. Leal. 2013. Germination responses of the invasive Calatropis procera (Ait.) R. Br. (Apocynaceae): comparisons with seeds from two ecosystems in northeastern Brazil. An Acad Bras Cienc 85(3):1025-1034.

72. Lessa, I. C. M. \& H. G. Bergallo. 2012. Modelling the population control of the domestic cat: an example from an island in Brazil. Brazilian Journal of Biology 72(3):445-452.

73. Lourenço-de-Oliveira, R.; M. G Castro; M. A. H. Braks \& L. P. Lounibos. 2004. The invasion of urban forest by dengue vectors in Rio de Janeiro. Journal of Vector Ecology 29(1):94-100.

74. Machado, I. F; L. F. B. Moreira \& L. Maltchik. 2012. Effects of pine invasion on anurans assemblage in southern Brazil coastal ponds. Amphibia-Reptilia 33(2):227-237.

75. Machado, T. D.; A. M. Sole-Cava; J. R. David \& B. C. Bitner-Mathe. 2005. Allozyme variability in an invasive drosophilid, Zaprionus indianus (Diptera: Drosophilidae): comparison of a recently introduced Brazilian population with Old World populations. Annales De La Societe Entomologique De France 41(1): 7-13.

76. Marichal, R; A. F. Martinez; C. Praxedes; D. Ruiz; A. F. Carvajal et al. 2010. Invasion of Pontoscolex corethrurus (Glossoscolecidae, Oligochaeta) in landscapes of the Amazonian deforestation arc. Applied Soil Ecology 46(3):443-449.

77. Marinho, M. S. \& H. S. Miranda. 2013. Efeito do fogo anual na mortalidade e no banco de sementes de Andropogon gayanus Kunth. no Parque Nacional de Brasília/DF. Biodiversidade Brasileira 3(2):149-158.

78. Martins, C. R.; J. D. V. Hay \& R. Carmona. 2009. Potencial invasor de duas cultivares de Melinis minutiflora no Cerrado brasileiro - características de sementes e estabelecimento de plântulas. Revista Árvore 33(4):713-722.
79. Martins, C. R.; J. D. V. Hay; B. M. T. Walter; C. E. B. Proença \& L. J. Vivaldi. 2011. Impact of invasion and management of molasses grass (Melinis minutiflora) on the native vegetation of the Brazilian Savanna. Brazilian Journal of Botany 34(1):73-90.

80. Melo-Silva, C.; M. P. Peres; J. N. Mesquita Neto; B. B. Gonçalves \& I. A. B. Leal. 2014. Biologia reprodutiva de L. leucocephala (Lam.) R. de Wit (Fabaceae: Mimosoideae): sucesso de uma espécie invasora. Neotropical Biology and Conservation 9(2):91-97.

81. Mengardo, A. L. \& V. R. Pivello. 2012. Phenology and fruit traits of Archontophoenix cunninghamiana, an invasive palm tree in the Atlantic Forest of Brazil. Ecotropica 18(1):45-54.

82. Mengardo, A. L. T.; C. L. Figueiredo; L. R. Tambosi \& V. R. Pivello. 2012. Comparing the establishment of an invasive and an endemic palm species in the Atlantic rainforest. Plant Ecology \& Diversity 5(3):345-354.

83. Mengardo, A. L. T. \& V. R. Pivello. 2014. The effects of an exotic palm on a native palm during the first demographic stages: contributions to ecological management. Acta Botanica Brasilica 28(4):552-558.

84. Modesto, T. C. \& H. G. Bergallo. 2008. Different environment, different amount of time spent in activities: the case of two mixed groups of the exotic Callithrix spp. at Ilha Grande, RJ, Brazil. Neotropical Biology and Conservation 3(3):112-118.

85. Moro, M. F.; C. Westerkamp \& F. R. Martins. 2013. Naturalization and potential impact of the exotic tree Azadirachta indica A.Juss. in Northeastern Brazil. Check List 9(1):153-156.

86. Nascimento, C. E. S.; M. Tabarelli; C. A. D. Silva; I. R. Leal; WdS Tavares et al. 2014. The introduced tree Prosopis juliflora is a serious threat to native species of the Brazilian Caatinga vegetation. Science of the Total Environment 481(108-113.

87. Nascimento, M. T.; R. M. Araújo; M. L. Dan; E. B. Fagundes Netto \& J. M. A. Braga. 2013. The Imperial Palm (Roystonea oleracea (Jacq.) O. F. Cook) as an invasive species of a wetland in Brazilian Atlantic forest. Wetlands ecology and management 21(5):367-371.

88. Ohlweiler, F. P.; M. C. A. Guimarães; F. Y. Takahashi \& J. M. Eduardo. 2010. Current distribution of Achatina fulica, in the state of São Paulo including records of Aelurostrongylus abstrusus (nematoda) larvae infestation. Revista do Instituto de Medicina Tropical de São Paulo 52(4):211-214.

89. Oliveira, L. C. \& C. E. V. Grelle. 2012. Introduced primate species of an Atlantic Forest region in Brazil: 
present and future implications for the native fauna. Tropical Conservation Science 5(1):112-120.

90. Oliveira, L. S. B.; L. A. Andrade; J. R. Fabricante \& G. S. Gonçalves. 2012. Structure of a Prosopis juliflora (Sw.) DC. population established in a temporary riverbed in the microregion of Cariri in the state of Paraíba. Semina: Ciências Agrárias 33(5):1769-1778.

91. Oliveira-Santos, L. G. R.; R. M. Dorazio; W. M. Tomas; G. Mourao \& F. A. S. Fernandez. 2011. No evidence of interference competition among the invasive feral pig and two native peccary species in a Neotropical wetland. Journal of Tropical Ecology 27(557-561.

92. Pegado, CuMA; L. A. Andrade; L. P. Félix \& I. M. Pereira. 2006. Efeitos da invasão biológica de algaroba - Prosopis juliflora (Sw.) DC. sobre a composição e a estrutura do estrato arbustivo-arbóreo da caatinga no município de Monteiro, PB, Brasil. Acta Botanica Brasilica 20(4):887-898.

93. Pellens, R. \& P. Grandcolas. 2002. Are successful colonizers necessarily invasive species? The case of the so-called "invading parthenogenetic cockroach", Pycnoscelus surinamensis, in the Brazilian Atlantic Forest. Revue d'écologie 57(3-4):253-261.

94. Pereira, D. G.; M. E. A. Oliveira \& C. R. Ruiz-Miranda. 2008. Interações entre calitriquídeos exóticos e nativos no Parque Nacional da Serra dos Órgãos-RJ. Revista Espaço e Geografia 11(1):87-114.

95. Petenon, D. \& V. R. Pivello. 2008. Invasive plants: representativeness of research from tropical countries in the global context. Nat Conservacao 6(1):183-195.

96. Pivello, V. R.; V. M. C. Carvalho; P. F. Lopes; A. A. Peccinini \& S Rosso. 1999a. Abundance and distribution of native and alien grasses in a "cerrado" (Brazilian savanna) biological reserve. Biotropica 31(1):71-82.

97. Pivello, V. R.; C. N. Shida \& S. T. Meirelles. 1999 b. Alien grasses in Brazilian savannas: a threat to the biodiversity. Biodiversity and Conservation 8(9):12811294.

98. Plucênio, R. M.; M. S. Dechoum \& T. T. Castellani. 2013. Invasão biológica em restinga: o estudo de caso de Terminalia catappa L. (Combretaceae). Biodiversidade Brasileira 3(2): 118-136.

99. Portela, R. C. Q.; D. M. S. Matos; L. P. de Siqueira; M. I. G Braz; L. Silva-Lima et al. 2009. Variation in aboveground biomass and necromass of two invasive species in the Atlantic rainforest, Southeast Brazil. Acta Botanica Brasilica 23(2):571-577.

100. Raíces, D. S. L.; F. S. Pessôa; J. L. Luz; T. JordãoNogueira; C. E. L. Esbérard et al. 2008. Feeding behaviour of the bat Phyllostomus hastatus (Pallas
1767) in jackfruit Artocarpus heterophyllus Lamarck (Moraceae), in Ilha Grande, Rio de Janeiro State, Brazil. Revista Brasileira de Zoociências 10(3):265-267.

101. Rangel, C. H. \& C. H. M. B. Neiva. 2013. Predação de vertebrados por cães Canis lupus F. Familiaris (Mammalia: Carnivora) no Jardim Botânico do Rio de Janeiro. Biodiversidade Brasileira 3(2):261-269.

102. Rangel, E. S. \& M. T. Nascimento. 2011. Occurrence of Calotropis procera (Ait.) R. Br. (Apocynaceae) as an invasive species in restinga vegetation. Acta Botanica Brasilica 25(3):657-663.

103. Ribeiro, M. O. \& A. S. Zaú. 2007. Levantamento populacional e manejo da exótica invasora Dracaena fragrans (L.) Ker-Gawl (Angiospermae-Liliaceae), em um trecho de Floresta Atlântica sob efeitos de borda no Parque Nacional da Tijuca, Rio de Janeiro, RJ. Revista Brasileira de Biociências 5(1):21-23.

104. Rocha, C. F. D.; L. A. Anjos \& H. G. Bergallo. 2011. Conquering Brazil: the invasion by the exotic gekkonid lizard Hemidactylus mabouia (Squamata) in Brazilian natural environments. Zoologia 28(6):747-754.

105. Rocha-Miranda, F.; M. J. Martins Silva \& A. F. Mendonça. 2006. First occurrence of bull frogs (Rana catesbeiana) in Federal District, central Brazil. Journal 1-4.

106. Rodolfo, A. M.; J. F. Cândido Jr.; L. G. Temponi \& M. Z. Gregorini. 2008. Citrus aurantium L.(laranja-apepu) e Hovenia dulcis Thunb.(uva-do-japão): espécies exóticas invasoras da trilha do Poço Preto no Parque Nacional do Iguaçu, Paraná, Brasil. Revista Brasileira de Biociências 6(S1):16-18.

107. Rossi, R. D.; C. R. Martins; P. L. Viana; E. L. Rodrigues \& J. E. C Figueira. 2014. Impact of invasion by molasses grass (Melinis minutiflora P. Beauv.) on native species and on fires in areas of campo-cerrado in Brazil. Acta Botanica Brasilica 28(4):631-637.

108. Sampaio, A. B.; H. G. Bergallo; K. T. Ribeiro; J. D. V. Hay \& R. Tidon. 2013. Diagnóstico e controle de espécies exóticas invasoras em áreas protegidas. Biodiversidade Brasileira 3(2):1-3.

109. Sampaio, A. B. \& I. B. Schmidt. 2013. Espécies exóticas invasoras em unidades de conservação federais do Brasil. Biodiversidade Brasileira 3(2):32-49.

110. Santilli, C. \& G. Durigan. 2014. Do alien species dominate plant communities undergoing restoration? A case study in the Brazilian savanna. Scientia Forestalis 42(103):371-382.

111. Santos, G. M. D.; C. M. L. Aguiar; J. Genini; C. F. Martins; F. C. V. Zanella et al. 2012. Invasive Africanized honeybees change the structure of native 
pollination networks in Brazil. Biological Invasions 14(11):2369-2378.

112. Santos, L. L.; A. L. B. Nascimento; F. J. Vieira; V. A. Silva; R. Voeks et al. 2014. The cultural value of invasive species: a case study from semi-arid northeastern Brazil. Economic Botany 68(3):283-300.

113. Sarmento, A. S. M.; C. Barbosa; T. T. Castellani \& N. Hanazaki. 2013. Interferência humana no estabelecimento e distribuição de Furcraea foetida (L.) Haw (Agavaceae) na Praia Mole, Ilha de Santa Catarina, Brasil: uma interface entre etnobotânica e espécies exóticas invasoras. Biodiversidade Brasileira 3(2):175-191.

114. Sarmento, R.; D. Brito; R. J. Ladle; G. D. Leal \& M. A. Efe. 2014. Invasive house (Rattus rattus) and brown rats (Rattus norvegicus) threaten the viability of redbilled tropicbird (Phaethon aethereus) in Abrolhos National Park, Brazil. Tropical Conservation Science 7(4):614-627.

115. Sato, M. N.; H. S. Miranda; S. S. Aires \& F. S. Aires. 2013. Alterações na fitossociologia do estrato rasteiro de uma área de campo sujo, invadida por Melinis minutiflora P. Beauv., submetida a corte anual. Biodiversidade Brasileira 3(2):137-148.

116. Silva, E. T. \& O. P. Ribeiro Filho. 2009. Predation on juveniles of the invasive american bullfrog Lithobates catesbeianus (Anura, Ranidae) by native frog and snake species in south-eastern Brazil. Herpetology Notes 2(215-218.

117. Silva, E. T.; O. P. Ribeiro Filho \& R. N. Feio. 2011. Predation of native anurans by invasive bullfrogs in southeastern Brazil: spatial variation and effect of microhabitat use by prey. South American Journal of Herpetology 6(1):1-10.

118. Silva, N. M.; C. C. Fantinel; V. L. Valente \& V. H. Valiati. 2005. Population dynamics of the invasive species Zaprionus indianus (Gupta) (Diptera: Drosophilidae) in communities of drosophilids of Porto Alegre city, southern of Brazil. Neotropical Entomology 34(3):363-374.

119. Silva, R. R.; F. T. A. Coelho; M. A. Anjos \& V. Vaz Filho. 2013. Controle do capim-gordura nas áreas de recuperação ambiental da Mineração Corumbaense Reunida (MCR), Corumbá, MS. Biodiversidade Brasileira 3(2):237-242.

120. Silva, Ú. S. R. \& D. M. S. Matos. 2006. The invasion of Pteridium aquilinum and the impoverishment of the seed bank in fire prone areas of Brazilian Atlantic Forest. Biodiversity \& Conservation 15(9):3035-3043.

121. Silverio, D. V.; P. M. Brando; J. K. Balch; F. E. Putz; D. C. Nepstad et al. 2013. Testing the Amazon savannization hypothesis: fire effects on invasion of a neotropical forest by native cerrado and exotic pasture grasses. Philosophical Transactions of the Royal Society B-Biological Sciences 368(1619):

122. Simberloff, D.; M. A. Nunez; N. J. Ledgard; A. Pauchard; D. M. Richardson et al. 2010. Spread and impact of introduced conifers in South America: lessons from other southern hemisphere regions. Austral Ecology 35(5):489-504.

123. Simões, K. C. C.; J. D. V. Hay; C. O. Andrade; O. A. Carvalho Júnior \& R. A. T. Gomes. 2013. Distribuição de cana-do-reino (Arundo donax L.) no Distrito Federal, Brasil. Biodiversidade Brasileira 3(97-105.

124. Soares, D. J. \& R. W. Barreto. 2008. Fungal pathogens of the invasive riparian weed Hedychium coronarium from Brazil and their potential for biological control. Fungal Diversity 28(85-96.

125. Sobrinho, M. S.; G. M. Tabatinga; I. C. Machado \& A. V. Lopes. 2013. Reproductive phenological pattern of Calotropis procera (Apocynaceae), an invasive species in Brazil: annual in native areas; continuous in invaded areas of caatinga. Acta Botanica Brasilica 27(2):456459.

126. Souza, A.; C. Z. Sandrin; M. F. Calio; S. T. Meirelles; V. R. Pivello et al. 2010. Seasonal variation of soluble carbohydrates and starch in Echinolaena inflexa, a native grass species from the Brazilian savanna, and in the invasive grass Melinis minutiflora. Brazilian Journal of Biololy 70(2):395-404.

127. Sponchiado, J.; G. L. Melo \& N. C. Caceres. 2011. First record of the invasive alien species Axis axis (Erxleben, 1777) (Artiodactyla: Cervidae) in Brazil. Biota Neotropica 11(3):403-406.

128. Thiengo, S. C.; F. A. Faraco; N. C. Salgado; R. H. Cowie \& MA Fernandez. 2007. Rapid spread of an invasive snail in South America: the giant African snail, Achatina fulica, in Brasil. Biological Invasions 9(6):693-702.

129. Tidon, R.; D. F. Leite \& B. F. D. Leao. 2003. Impact of the colonisation of Zaprionus (Diptera, Drosophilidae) in different ecosystems of the Neotropical region: 2 years after the invasion. Biological Conservation 112(3):299-305.

130. Tortato, M. A.; R. F. Bressan \& T. S. Kunz. 2014. Reproduction of two exotic species of Trachemys Agassiz, 1857 (Testudines, Emydidae) at Parque Estadual da Serra do Tabuleiro, state of Santa Catarina, southern Brazil. Herpetology Notes 7(11-15.

131. Vasconcelos, S. D. \& R. L. Salgado. 2014. First record of six Calliphoridae (Diptera) species in a seasonally dry 
tropical forest in Brazil: evidence for the establishment of invasive species. Florida Entomologist 97(2):814816.

132. Vigilato, G. R. \& R. Zampar. 2011. Susceptibilidade das zonas de recuperação de uma unidade de conservação à invasão biológica por espécies arbóreas exóticas. SaBios-Revista de Saúde e Biologia 6(3):

133. Vilela, C. R. \& L. Mori. 2014. The invasive spotted-wing Drosophila (Diptera, Drosophilidae) has been found in the city of São Paulo (Brazil). Revista Brasileira De Entomologia 58(4):371-375.

134. Voltolini, J. C. \& L. Zanco. 2010. Densidade de plântulas e jovens de espécies nativas de Floresta Atlântica em áreas com e sem o pinheiro americano (Pinus elliottii). Revista Biociências 16(2):102-108.

135. Wilson, J. R. U.; P. Caplat; I. A. Dickie; C. Hui; B. D. Maxwell et al. 2014. A standardized set of metrics to assess and monitor tree invasions. Biological Invasions 16(3):535-551.

136. Wilson, J. R. U.; C. Gairifo; M. R. Gibson; M. Arianoutsou; B. B. Bakar et al. 2011. Risk assessment, eradication, and biological control: global efforts to limit Australian acacia invasions. Diversity and Distributions 17(5):1030-1046.

137. Zanol, J.; M. A. Fernandez; A. P. M. Oliveira; C. A. D. Russo \& SC Thiengo. 2010. The exotic invasive snail Achatina fulica (Stylommatophora, Mollusca) in the State of Rio de Janeiro (Brazil): current status. Biota Neotropica 10(3):447-451.
138. Zenni, R. D. 2014. Analysis of introduction history of invasive plants in Brazil reveals patterns of association between biogeographical origin and reason for introduction. Austral Ecology 39(4):401-407.

139. Zenni, R. D.; J. K. Bailey \& D. Simberloff. 2014. Rapid evolution and range expansion of an invasive plant are driven by provenance-environment interactions. Ecology Letters 17(6):727-735.

140. Zenni, R. D. \& D. Simberloff. 2013. Number of source populations as a potential driver of pine invasions in Brazil. Biological Invasions 15(7):1623-1639.

141. Zenni, R. D. \& S. R. Ziller. 2011. An overview of invasive plants in Brazil. Brazilian Journal of Botany 34(3):431.

142. Ziller, S. R. \& M. S. Dechoum. 2013. Plantas e vertebrados exóticos invasores em unidades de conservação no Brasil. Biodiversidade Brasileira 3(2):4-31.

143. Ziller, S. R. \& S. Zalba. 2007. Proposals to prevent and control exotic invasive species. Nat Conservacao 5(2):78-85. 


\section{ANEXO 2}

Relação de instrumentos legais específicos e que fazem menção a espécies exóticas invasoras nas esferas federal, estadual e municipal no Brasil. As normativas estão apresentadas por esfera, tipo e órgão responsável e em ordem cronológica decrescente. *Normativas consideradas específicas sobre o tema (Fonte: Instituto Hórus, 2015).

\begin{tabular}{|c|c|c|}
\hline \multicolumn{3}{|c|}{ Esfera Federal } \\
\hline Tipo e órgão responsável & $\begin{array}{l}\text { Número e data de } \\
\text { publicação }\end{array}$ & De que trata \\
\hline \multicolumn{3}{|l|}{ Leis } \\
\hline $\begin{array}{l}\text { Congresso Nacional/ } \\
\text { Presidência da República }\end{array}$ & \begin{tabular}{|l|} 
Lei no 12.651, de 25 de \\
maio de 2012 \\
\end{tabular} & Dispõe sobre a proteção da vegetação nativa \\
\hline $\begin{array}{l}\text { Congresso Nacional/ } \\
\text { Presidência da República }\end{array}$ & $\begin{array}{l}\text { Lei no } 9.985 \text {, de } \\
\text { 18/07/2000 }\end{array}$ & $\begin{array}{l}\text { Regulamenta o art. 225, § } 1^{\circ} \text {, incisos I, II, III e VII da } \\
\text { Constituição Federal, institui o Sistema Nacional de Unidades } \\
\text { de Conservação da Natureza e dá outras providências. }\end{array}$ \\
\hline $\begin{array}{l}\text { Congresso Nacional/ } \\
\text { Presidência da República }\end{array}$ & $\begin{array}{l}\text { Lei no 9.605, de } \\
12 / 02 / 1998\end{array}$ & Institui a Lei de Crimes Ambientais. \\
\hline \multicolumn{3}{|l|}{ Decretos } \\
\hline Presidência da República & $\begin{array}{l}\text { Decreto } n^{\circ} 6.514 \text {, de } \\
22 / 06 / 2008\end{array}$ & $\begin{array}{l}\text { Dispõe sobre as infrações e sanções administrativas ao meio } \\
\text { ambiente, estabelece o processo administrativo federal para } \\
\text { apuração destas infrações, e dá outras providências. }\end{array}$ \\
\hline Presidência da República & \begin{tabular}{|l|} 
Decreto $\mathrm{n}^{\mathrm{o}} 6.660$, de \\
$21 / 11 / 2008$ \\
\end{tabular} & $\begin{array}{l}\text { Dispõe sobre a utilização e proteção da vegetação nativa do } \\
\text { Bioma Mata Atlântica. }\end{array}$ \\
\hline Presidência da República & $\begin{array}{l}\text { Decreto } n^{\circ} 4.339, \text { de } \\
22 / 08 / 2002\end{array}$ & Institui a Política Nacional de Biodiversidade. \\
\hline Presidência da República & $\begin{array}{l}\text { Decreto no } 4.340, \text { de } \\
22 / 08 / 2002\end{array}$ & $\begin{array}{l}\text { Regulamenta o Sistema Nacional de Unidades de Conservação } \\
\text { (SNUC). }\end{array}$ \\
\hline Presidência da República & $\begin{array}{l}\text { Decreto } \mathrm{n}^{\mathrm{o}} 2.519, \text { de } \\
16 / 03 / 1998\end{array}$ & Promulga a Convenção sobre Diversidade Biológica. \\
\hline Congresso Nacional & $\begin{array}{l}\text { Decreto Legislativo } \mathrm{n}^{\mathrm{o}} \\
2 \text {, de } 03 / 02 / 1994\end{array}$ & Aprova o texto da Convenção sobre Diversidade Biológica. \\
\hline \multicolumn{3}{|l|}{ Deliberações } \\
\hline $\begin{array}{l}\text { Comissão Nacional da } \\
\text { Biodiversidade (CONABIO) }\end{array}$ & \begin{tabular}{|l|} 
Deliberação CONABIO \\
no 52, de 25/06/2008 \\
\end{tabular} & $\begin{array}{l}\text { Dispõe sobre propostas para a produção sustentável de } \\
\text { biocombustíveis considerando a biodiversidade. }\end{array}$ \\
\hline CONABIO & \begin{tabular}{|l|} 
Deliberação CONABIO \\
no 49, de 30/08/2006*
\end{tabular} & $\begin{array}{l}\text { Criação da Câmera Técnica Permanente sobre Espécies } \\
\text { Exóticas Invasoras e designação das instituições envolvidas. }\end{array}$ \\
\hline \multicolumn{3}{|l|}{ Instruções normativas } \\
\hline $\begin{array}{l}\text { Instituto Brasileiro do Meio } \\
\text { Ambiente e dos Recursos } \\
\text { Naturais Renováveis (IBAMA) }\end{array}$ & $\begin{array}{l}\text { Instrução Normativa no } \\
3 \text {, de } 31 / 01 / 2013^{*}\end{array}$ & Decreta a nocividade do javali e dispõe sobre seu controle. \\
\hline IBAMA & \begin{tabular}{|l|} 
Instrução Normativa \\
no 7 , de $02 / 07 / 2012^{*}$
\end{tabular} & $\begin{array}{l}\text { Dispõe sobre o registro de herbicidas para controle de espécies } \\
\text { exóticas invasoras em unidades de conservação. }\end{array}$ \\
\hline $\begin{array}{l}\text { Ministério do Meio Ambiente } \\
\text { (MMA) }\end{array}$ & $\begin{array}{l}\text { Instrução Normativa no } \\
5 \text {, de } 8 / 09 / 2009\end{array}$ & $\begin{array}{l}\text { Dispõe sobre os procedimentos metodológicos para restauração } \\
\text { e recuperação das Áreas de Preservação Permanente e da } \\
\text { Reserva Legal instituídas pela Lei no } 4.771 \text {, de } 15 \text { de setembro } \\
\text { de } 1965 \text {. }\end{array}$ \\
\hline IBAMA & $\begin{array}{l}\text { Instrução Normativa nº } \\
203 \text { de } 22 / 10 / 2008\end{array}$ & $\begin{array}{l}\text { Dispõe sobre normas, critérios e padrões para a explotação com } \\
\text { finalidade ornamental e de aquariofilia de peixes nativos ou } \\
\text { exóticos de águas continentais. }\end{array}$ \\
\hline IBAMA & \begin{tabular}{|l|} 
Instrução Normativa ${ }^{\circ}{ }^{\circ}$ \\
141, de $19 / 12 / 2006$ \\
\end{tabular} & Regulamenta o manejo e controle da fauna sinantrópica nociva. \\
\hline IBAMA & $\begin{array}{l}\text { Instrução Normativa } n^{o} \\
73 \text {, de } 18 / 08 / 2005^{*}\end{array}$ & $\begin{array}{l}\text { Proíbe, em todo o território brasileiro, a criação e comercialização } \\
\text { de moluscos terrestres da espécie Achatina fulica. }\end{array}$ \\
\hline
\end{tabular}




\begin{tabular}{|c|c|c|}
\hline \multicolumn{3}{|l|}{ Portarias } \\
\hline IBAMA & $\begin{array}{l}\text { Portaria } \mathrm{n}^{\circ} 14, \text { de } 26 \text { de } \\
\text { maio de } 2010^{*}\end{array}$ & Autoriza o uso emergencial de herbicidas em áreas naturais. \\
\hline IBAMA & \begin{tabular}{|l|} 
Portaria no 93, de \\
$07 / 07 / 1998$ \\
\end{tabular} & $\begin{array}{l}\text { Apresenta a lista de animais considerados domésticos para fins } \\
\text { de operacionalização do IBAMA. }\end{array}$ \\
\hline IBAMA & $\begin{array}{l}\text { Portaria } n^{0} 145, \text { de } \\
\text { 29/10/1998 }\end{array}$ & $\begin{array}{l}\text { Estabelece normas para a introdução, reintrodução e } \\
\text { transferência de peixes, crustáceos, moluscos e macrófitas } \\
\text { aquáticas para fins de aquicultura, excluindo-se as espécies } \\
\text { animais ornamentais. }\end{array}$ \\
\hline IBAMA & $\begin{array}{l}\text { Portaria } n^{0} 142, \text { de } \\
\text { 22/12/1994 }\end{array}$ & $\begin{array}{l}\text { Proíbe a introdução, a transferência, o cultivo e a comercialização } \\
\text { de bagre africano (Clarias gariepinus) e bagre-do-canal } \\
\text { (Ictalurus punctatus) nas áreas abrangidas pelas bacias dos rios } \\
\text { Amazonas e Paraguai. }\end{array}$ \\
\hline Ministério da Agricultura & \begin{tabular}{|l|} 
Portaria $n^{\circ} 205$, de \\
$13 / 03 / 1979$ \\
\end{tabular} & $\begin{array}{l}\text { Proíbe o comércio, a importação, a exportação e o transporte de } \\
\text { Eragrostis plana (capim-annoni 2). }\end{array}$ \\
\hline \multicolumn{3}{|l|}{ Resoluções } \\
\hline CONAMA & $\begin{array}{l}\text { Resolução no 429, de } \\
\text { 28/02/2011 }\end{array}$ & $\begin{array}{l}\text { Dispõe sobre a metodologia de recuperação de áreas de } \\
\text { preservação permanente (APP). }\end{array}$ \\
\hline CONABIO & $\begin{array}{l}\text { Resolução } n^{0} 5 \\
21 / 10 / 2009^{*} \\
\end{array}$ & $\begin{array}{l}\text { Dispõe sobre a Estratégia Nacional para Espécies Exóticas } \\
\text { Invasoras. }\end{array}$ \\
\hline CONAMA & $\begin{array}{l}\text { Resolução no 237, de } \\
\text { 19/12/1997 }\end{array}$ & Dispõe sobre critérios para licenciamento ambiental. \\
\hline \multicolumn{3}{|c|}{ Esfera Estadual } \\
\hline $\begin{array}{l}\text { Tipo e estado/ órgão } \\
\text { responsável }\end{array}$ & $\begin{array}{l}\text { Número e data de } \\
\text { publicação }\end{array}$ & De que trata \\
\hline \multicolumn{3}{|l|}{ Leis } \\
\hline Rio Grande do Sul & $\begin{array}{l}\text { Lei no } 13.187, \text { de } \\
\text { 23/06/2009* }\end{array}$ & $\begin{array}{l}\text { Institui o Programa de controle de capim-annoni (Eragrostis } \\
\text { plana) no estado do Rio Grande do Sul. }\end{array}$ \\
\hline Pernambuco & $\begin{array}{l}\text { Lei no } 13.787 \text {, de } \\
\text { 08/06/2009 }\end{array}$ & $\begin{array}{l}\text { Institui o Sistema Estadual de Unidades de Conservação da } \\
\text { Natureza - SEUC, no âmbito do Estado de Pernambuco, e dá } \\
\text { outras providências . }\end{array}$ \\
\hline Santa Catarina & $\begin{array}{l}\text { Lei no } 14.675, \text { de } \\
13 / 04 / 2009\end{array}$ & Institui o Código Estadual do Meio Ambiente de Santa Catarina. \\
\hline \multicolumn{3}{|l|}{ Decretos } \\
\hline São Paulo & $\begin{array}{l}\text { Decreto no } 53.939, \text { de } \\
\text { 06/01/2009 }\end{array}$ & $\begin{array}{l}\text { Dispõe sobre a manutenção, recomposição, condução da } \\
\text { regeneração natural, compensação e composição da área de } \\
\text { Reserva Legal de imóveis rurais no estado de São Paulo e dá } \\
\text { providências. }\end{array}$ \\
\hline \multicolumn{3}{|l|}{ Deliberações } \\
\hline $\begin{array}{l}\text { São Paulo/Conselho } \\
\text { Estadual de Meio Ambiente } \\
\text { (CONSEMA) }\end{array}$ & $\begin{array}{l}\text { Deliberação no } 30, \text { de } \\
\text { 09/11/2011* }\end{array}$ & $\begin{array}{l}\text { Reconhece lista de espécies exóticas com potencial de } \\
\text { bioinvasão no estado de São Paulo. }\end{array}$ \\
\hline São Paulo/CONSEMA & $\begin{array}{l}\text { Deliberação Normativa } \\
\text { no } 2 \text {, de } 09 / 11 / 2011^{*}\end{array}$ & $\begin{array}{l}\text { Dispõe sobre a elaboração e a atualização de lista de espécies } \\
\text { exóticas com potencial de bioinvasão no estado de São Paulo e } \\
\text { dá outras providências. }\end{array}$ \\
\hline \multicolumn{3}{|l|}{ Instruções normativas } \\
\hline $\begin{array}{l}\text { Rio Grande do Sul/ } \\
\text { Secretaria do Ambiente e } \\
\text { Desenvolvimento Sustentável } \\
\text { (SEMA) }\end{array}$ & $\begin{array}{l}\text { Instrução Normativa no } \\
09 \text {, de } 10 / 12 / 2014^{*}\end{array}$ & $\begin{array}{l}\text { Estabelece procedimentos para o uso de Acacia mearnsii } \\
\text { (acácia-negra), enquadrada na Categoria } 2 \text { da Portaria SEMA } \\
\text { no }^{-79 / 2013 .}\end{array}$ \\
\hline Rio Grande do Sul/SEMA & $\begin{array}{l}\text { Instrução Normativa } n^{\circ} \\
10 \text {, de } 10 / 12 / 2014^{*}\end{array}$ & $\begin{array}{l}\text { Estabelece procedimentos para a execução de medidas de } \\
\text { prevenção, controle e monitoramento referentes ao Artigo } 10 \\
\text { da Portaria SEMA no } 79 / 2013 \text {. }\end{array}$ \\
\hline
\end{tabular}




\begin{tabular}{|c|c|c|}
\hline Rio Grande do Sul/SEMA & $\begin{array}{l}\text { Instrução Normativa no } \\
11 \text {, de } 10 / 12 / 2014^{*}\end{array}$ & $\begin{array}{l}\text { Estabelece procedimentos para o uso de Urochloa spp. } \\
\text { (braquiárias), enquadrada na Categoria } 2 \text { da Portaria SEMA no } \\
\text { 79/2013. }\end{array}$ \\
\hline Rio Grande do Sul/SEMA & $\begin{array}{l}\text { Instrução Normativa no } \\
12 \text {, de } 10 / 12 / 2014^{*}\end{array}$ & $\begin{array}{l}\text { Estabelece procedimentos para o controle e a erradicação de } \\
\text { plantas exóticas invasoras enquadradas na Categoria } 1 \text { da } \\
\text { Portaria SEMA no } 79 / 2013 \text {. }\end{array}$ \\
\hline Rio Grande do Sul/SEMA & $\begin{array}{l}\text { Instrução Normativa no } \\
13 \text {, de } 10 / 12 / 2014^{*}\end{array}$ & $\begin{array}{l}\text { Estabelece procedimentos para o uso de Archontophoenix } \\
\text { cunninghamiana (palmeira-imperial), enquadrada na Categoria } \\
2 \text { da Portaria SEMA no } 79 / 2013 \text {. }\end{array}$ \\
\hline Rio Grande do Sul/SEMA & $\begin{array}{l}\text { Instrução Normativa no } \\
14, \text { de } 10 / 12 / 2014^{*}\end{array}$ & $\begin{array}{l}\text { Estabelece procedimentos para o uso de Pinus spp., enquadrado } \\
\text { na Categoria } 2 \text { da Portaria SEMA no } 79 / 2013 \text {. }\end{array}$ \\
\hline Rio Grande do Sul/SEMA & $\begin{array}{l}\text { Instrução Normativa }{ }^{\circ} \\
04 \text {, de } 11 / 11 / 2014^{*}\end{array}$ & $\begin{array}{l}\text { Estabelece o ordenamento e o controle das atividades que } \\
\text { envolvem a criação de peixes exóticos invasores. }\end{array}$ \\
\hline Rio Grande do Sul/SEMA & $\begin{array}{l}\text { Instrução Normativa no } \\
05 \text {, de } 11 / 11 / 2014^{*}\end{array}$ & $\begin{array}{l}\text { Estabelece o ordenamento e o controle das atividades que } \\
\text { envolvem a criação de Lithobates catesbeianus (rã-touro), espécie } \\
\text { enquadrada na Categoria } 2 \text { da Portaria SEMA no } 79 / 2013 \text {. }\end{array}$ \\
\hline \begin{tabular}{|l|} 
Espírito Santo/Instituto \\
Estadual de Meio Ambiente de \\
Recursos Hídricos \\
\end{tabular} & $\begin{array}{l}\text { Instrução Normativa no } \\
03 \text {, de } 05 / 02 / 2007^{*}\end{array}$ & $\begin{array}{l}\text { Normatiza o processo de eliminação e controle de espécies } \\
\text { vegetais exóticas invasoras em Unidades de Conservação sob } \\
\text { administração do IEMA-ES. }\end{array}$ \\
\hline Rio Grande do Sul/IBAMA & $\begin{array}{l}\text { Instrução Normativa no } \\
71 \text {, de } 04 / 08 / 2005^{*}\end{array}$ & $\begin{array}{l}\text { Autoriza o controle populacional do javali - Sus scrofa -, por } \\
\text { meio da captura e do abate, em todo o Estado do Rio Grande } \\
\text { do Sul. }\end{array}$ \\
\hline \multicolumn{3}{|l|}{ Portarias } \\
\hline Rio Grande do Sul/SEMA & Portaria no $79 / 2013^{*}$ & $\begin{array}{l}\text { Reconhece a Lista Oficial de Espécies Exóticas Invasoras do } \\
\text { Estado do Rio Grande do Sul. }\end{array}$ \\
\hline \begin{tabular}{|l|} 
Rio Grande do Sul/Secretaria \\
da Agricultura, Pecuária, Pesca \\
e Agronegócio (SEAPPA) \\
\end{tabular} & Portaria no $093 / 2011^{*}$ & Estabelece medidas de controle ambiental de javali no estado. \\
\hline Rio Grande do Sul/SEAPPA & Portaria $\mathrm{n}^{\mathrm{o}} 183 / 2010^{*}$ & Regulamenta a caça e o controle do javali no estado. \\
\hline $\begin{array}{l}\text { Santa Catarina/Batalhão de } \\
\text { Polícia Militar Ambiental }\end{array}$ & Portaria $\mathrm{n}^{\mathrm{o}} 004 / 2010^{*}$ & Define normas para controle de javali no estado. \\
\hline $\begin{array}{l}\text { Paraná /Instituto Ambiental do } \\
\text { Paraná (IAP) }\end{array}$ & $\begin{array}{l}\text { Portaria } n^{-} 125, \text { de } \\
07 / 08 / 2009^{*}\end{array}$ & $\begin{array}{l}\text { Reconhece a Lista Oficial de Espécies Exóticas Invasoras para } \\
\text { o Estado do Paraná, estabelece normas de controle e dá outras } \\
\text { providências. }\end{array}$ \\
\hline Paraná /IAP & $\begin{array}{l}\text { Portaria } n^{\circ} 019, \text { de } \\
17 / 02 / 2009^{*}\end{array}$ & Institui o Comitê Estadual de Espécies Exóticas Invasoras. \\
\hline Paraná /IAP & $\begin{array}{l}\text { Portaria } n^{0} 176, \text { de } \\
\text { 19/09/2007* } \\
\end{array}$ & $\begin{array}{l}\text { Regulamenta o corte de espécies florestais exóticas em } \\
\text { perímetro urbano. }\end{array}$ \\
\hline Paraná /IAP & $\begin{array}{l}\text { Portaria } n^{\circ} 096, \text { de } \\
\text { 22/05/2007* }\end{array}$ & $\begin{array}{l}\text { Isenta a matéria prima florestal exótica da obrigatoriedade } \\
\text { de reposição florestal, da prévia aprovação para exploração e } \\
\text { transporte. }\end{array}$ \\
\hline Paraná /IAP & $\begin{array}{l}\text { Portaria } n^{0} 192, \text { de } \\
02 / 12 / 2005^{*}\end{array}$ & $\begin{array}{l}\text { Normatiza o processo de eliminação e controle de espécies } \\
\text { vegetais exóticas invasoras em unidades de conservação de } \\
\text { proteção integral sob administração do IAP. }\end{array}$ \\
\hline Rio Grande do Sul/SEMA & $\begin{array}{l}\text { Portaria } \mathrm{n}^{\mathbf{0}} 98 \text {, de } \\
\text { 01/12/1993* }\end{array}$ & $\begin{array}{l}\text { Proíbe a introdução em ambientes naturais ou artificiais, o } \\
\text { cultivo, a comercialização e o transporte da bagres africanos } \\
\text { (família Clariidae). }\end{array}$ \\
\hline \multicolumn{3}{|l|}{ Resoluções } \\
\hline Santa Catarina/CONSEMA & $\begin{array}{l}\text { Resolução } n^{\circ} \text { 8, de } 14 \text { de } \\
\text { setembro de 2012* }\end{array}$ & $\begin{array}{l}\text { Reconhece a Lista Oficial de Espécies Exóticas Invasoras no } \\
\text { Estado de Santa Catarina e dá outras providências. } \\
\end{array}$ \\
\hline São Paulo/SMA & $\begin{array}{l}\text { Resolução no } 33 \text {, de } \\
\text { 22/05/2009* }\end{array}$ & $\begin{array}{l}\text { Dispõe sobre a criação de Grupo de Trabalho para o } \\
\text { desenvolvimento de proposta para "Estratégia Estadual sobre } \\
\text { Espécies Exóticas Invasoras". }\end{array}$ \\
\hline
\end{tabular}




\begin{tabular}{|c|c|c|}
\hline Paraná - IBAMA, SEMA e IAP & $\begin{array}{l}\text { Resolução Conjunta no }{ }^{-} \\
002 / 2008\end{array}$ & $\begin{array}{l}\text { Estabelece normas e procedimentos para regularização } \\
\text { ambiental de tanques, viveiros, açudes, pequenos reservatórios } \\
\text { e lagoas destinados à produção de peixes em águas continentais } \\
\text { no estado do Paraná. }\end{array}$ \\
\hline Paraná/IAP & $\begin{array}{l}\text { Resolução nº } 151, \text { de } \\
\text { 08/12/1997* }\end{array}$ & Proíbe Tecoma stans como planta nociva no Estado do Paraná. \\
\hline \multicolumn{3}{|c|}{ ESFERA MUNICIPAL } \\
\hline $\begin{array}{l}\text { Tipo e município/ estado/ } \\
\text { órgão responsável }\end{array}$ & $\begin{array}{l}\text { Número e data de } \\
\text { publicação }\end{array}$ & De que trata \\
\hline \multicolumn{3}{|l|}{ Leis } \\
\hline Florianópolis/SC & $\begin{array}{l}\text { Lei Municipal } \mathrm{n}^{0} 9.097 \\
\text { de } 18 \text { de outubro de } \\
2012 *\end{array}$ & $\begin{array}{l}\text { Institui a política municipal de remoção e a substituição } \\
\text { de Pinus, Eucalyptus e Casuarina por espécies nativas no } \\
\text { município de Florianópolis. }\end{array}$ \\
\hline \multicolumn{3}{|l|}{ Decretos } \\
\hline Rio de Janeiro/RJ & $\begin{array}{l}\text { Decreto } \mathrm{n}^{\mathrm{o}} 33814 \text {, de } \\
18 / 05 / 2011 *\end{array}$ & $\begin{array}{l}\text { Estabelece o Programa Municipal do Rio de Janeiro de Controle } \\
\text { de Espécies Exóticas Invasoras. }\end{array}$ \\
\hline Bauru/SP & $\begin{array}{l}\text { Decreto no } 10.987 \text {, de } \\
16 / 07 / 2009 *\end{array}$ & $\begin{array}{l}\text { Declara a lista oficial das espécies exóticas invasoras ou } \\
\text { com potencial de invasão do Município de Bauru e dá outras } \\
\text { providências. }\end{array}$ \\
\hline Curitiba/PR & Decreto n- 473/2008* & $\begin{array}{l}\text { Estabelece a lista oficial de espécies florestais exóticas invasoras } \\
\text { para o município de Curitiba. }\end{array}$ \\
\hline \multicolumn{3}{|l|}{ Resoluções } \\
\hline $\begin{array}{l}\text { Rio de Janeiro/RJ/Secretaria } \\
\text { Municipal de Meio Ambiente }\end{array}$ & $\begin{array}{l}\text { Resolução SMAC no } \\
555 \text {, de } 03 \text { de abril de } \\
2014 *\end{array}$ & $\begin{array}{l}\text { Regulamente o Plano de controle de espécies animais exóticas } \\
\text { invasoras }\end{array}$ \\
\hline $\begin{array}{l}\text { Rio de Janeiro/RJ/Secretaria } \\
\text { Municipal de Meio Ambiente }\end{array}$ & $\begin{array}{l}\text { Resolução SMAC n⿳o } \\
544, \text { de } 28 \text { de março de } \\
2014 *\end{array}$ & Atualiza a lista de espécies vegetais exóticas invasoras \\
\hline $\begin{array}{l}\text { Rio de Janeiro/RJ/Secretaria } \\
\text { Municipal de Meio Ambiente }\end{array}$ & $\begin{array}{l}\text { Resolução SMAC no } \\
492, \text { de } 05 / 06 / 2011^{*}\end{array}$ & $\begin{array}{l}\text { Regulamenta Programa Municipal do Rio de Janeiro de } \\
\text { Controle de Espécies Exóticas Invasoras. }\end{array}$ \\
\hline $\begin{array}{l}\text { Bauru/SP/ Secretaria Municipal } \\
\text { de Meio Ambiente }\end{array}$ & $\begin{array}{l}\text { Resolução nº } 11 \text { de } \\
2007 *\end{array}$ & $\begin{array}{l}\text { Declara a lista oficial das espécies exóticas invasoras ou } \\
\text { com potencial de invasão do Município de Bauru e dá outras } \\
\text { providências. }\end{array}$ \\
\hline $\begin{array}{l}\text { Bauru/SP/ Secretaria Municipal } \\
\text { de Meio Ambiente }\end{array}$ & $\begin{array}{l}\text { Resolução nº } 08 \text { de } \\
2007^{*}\end{array}$ & $\begin{array}{l}\text { Aprova as Estratégias de Implementação do Plano de Manejo } \\
\text { do Caramujo-Africano no âmbito do Município de Bauru e dá } \\
\text { outras providências. }\end{array}$ \\
\hline \multicolumn{3}{|l|}{ Portarias } \\
\hline $\begin{array}{l}\text { São Paulo/SP/Secretaria } \\
\text { Municipal do Verde e do Meio } \\
\text { Ambiente (SVMA) }\end{array}$ & Portaria n ${ }^{\circ}$ 19/2010* & $\begin{array}{l}\text { Institui Lista Oficial de Espécies Vegetais Exóticas Invasoras } \\
\text { no município de São Paulo-SP. }\end{array}$ \\
\hline São Paulo/SP/SVMA & Portaria $\mathrm{n}^{\mathrm{o}}$ 154/2009* & $\begin{array}{l}\text { Disciplina as medidas visando à erradicação e ao controle de } \\
\text { espécies vegetais exóticas invasoras por Plano de Manejo e } \\
\text { institui a Lista Oficial de Espécies Vegetais Exóticas Invasoras } \\
\text { no município de São Paulo-SP. }\end{array}$ \\
\hline
\end{tabular}

